\title{
Interval observer-based fault detectability analysis using mixed set-invariance theory and sensitivity analysis approach
}

\author{
Masoud Pourasghar, Vicenç Puig and Carlos Ocampo-Martinez \\ Automatic Control Department, Universitat Politècnica de Catalunya, Institut de Robòtica i Informàtica \\ Industrial (CSIC-UPC), Supervision, Safety and Automatic Control Research Center (CS2AC), C/. \\ Llorens $i$ Artigas 4-6, 08028 Barcelona, Spain. (e-mail: \{mpourasgharlaf, vpuig, cocampo\}@iri.upc.edu)
}

\begin{abstract}
This paper addresses the characterization of the minimum detectable fault (MDF) by means of residual sensitivity integrated with the set-invariance theory when using an interval observer-based approach as a Fault Detection (FD) scheme. Uncertainties (disturbances and noise) are considered as of unknown but bounded nature (i.e., in the set-membership framework). A zonotopic-set representation towards reducing set operations to simple matrix calculations is utilized to bound the state/output estimations provided by the interval observer-based approach. In order to show the connection between sensitivity and setinvariance analyses, mathematical expressions of the MDF are derived when considering different types of faults. Finally, a simulation case study based on a quadruple-tank system is employed to both illustrate and discuss the effectiveness of the proposed approach. Interval observer-based FD scheme is used to test the MDF obtained from the integration of both residual sensitivity analysis and set-invariance theory in the considered case study.
\end{abstract}

Keywords: Fault detection, bounded uncertainties, interval observer-based approach, set-invariance approach, sensitivity analysis, zonotopes, minimum detectable fault

\section{Introduction}

Associated with the goal of increasing system performance as well as its safety and reliability, Fault Detection (FD) has received more and more attention from the scientific community over the past years. In particular, model-based FD is one of the most developed family of approaches (Chen \& Patton, 1999; Gertler, 1998). Moreover, the use of mathematical models to describe the system behaviour is known as the fundamental concept of model-based FD approaches (Cai, Ferdowsi, \& Sarangapani, 2016; Combastel, 2015; Gertler, 1998). Therefore, to improve performance of FD is directly related with the quality of the mathematical model (Blanke, Kinnaert, Lunze, Staroswiecki, \& Schröder, 2006; Chen \& Patton, 1999). But, in practice, a mismatch between the actual process and its mathematical model is non-negligible even if there are no process faults because of the presence of model uncertainty, unknown disturbances and noises, (Puig, Montes de Oca, \& Blesa, 2013). Thus, dealing with uncertainty and noise/disturbance is an important issue that implies developing robust model-based FD approaches (Chen \& Patton, 1999). In recent years, several methods have been developed and introduced to explicitly consider such uncertainties in the models. These methods can be classified into stochastic and deterministic classes of approaches. In the former class, uncertainties are represented using random variables, and in the latter class (also called set-based approaches), uncertainties are assumed unknown but bounded by means of different geometrical structures, e.g., interval boxes, polytopes, ellipsoids and zonotopes (Puig, 2010; Raïssi, Efimov, \& Zolghadri, 2012; Uusitalo, Lehikoinen, Helle, \& Myrberg, 2015). 
FD in model-based approaches relies on checking the consistency of the observed behavior from measured outputs using sensors and the estimated behavior that is computed by using the system model (Gertler, 1998). This consistency test is based on generating the residual by computing the difference between the output predicted values from the model and the real measured values obtained from the sensors (Chen \& Patton, 1999; Gertler, 2015). Then, the fault can be detected by comparing that residual with a threshold value that takes into account the uncertainty associated with measurement noise, disturbances, and the model mismatch between the real and modeled system behavior (Puig, Quevedo, Escobet, \& Stancu, 2003). If the residual is larger than such threshold, the existence of the fault can be proved (Fagarasan, Ploix, \& Gentil, 2004; Puig, 2010). Otherwise, the system is assumed to be still in healthy operation and working properly.

Methods that explicitly consider uncertainty in the FD task are known as robust approaches. Most robust approaches try to maximize the fault sensitivity while minimizing the sensitivity to uncertainty at the residual generation phase, following the active robust approach (Chen \& Patton, 1999; Ding, 2008; Zhong, Ding, Lam, \& Wang, 2003). On the other hand, the passive robust approach tries to bound the effect of uncertainty in the residual evaluation phase by generating adaptive thresholds (Puig, 2010). In the former class, the main idea is to decouple the effect of the uncertainty (Chen \& Patton, 2012), and the latter approach is based on enhancing the robustness of the FD system at the decision-making stage (Puig, Quevedo, Escobet, \& de las Heras, 2002). The main purpose of the passive FD approach is to determine whether or not there is any member in the uncertainty set that can explain the measurements. Then, any inconsistency between the measurement and this set is considered as fault occurrence. Therefore, it is not necessary to know the exact model of as a fault. In recent years, there has been an increasing interest in using the passive approach since its main advantage over the corresponding active approach is to achieve robustness in the FD procedure in spite of the number of uncertain parameters in the model by using the underlying parameter representation without any simplification (Chai \& Qiao, 2014; Silvestre, Rosa, Hespanha, \& Silvestre, 2017; Tabatabaeipour, 2015; Thabet, Combastel, Raïssi, \& Zolghadri, 2015; Xu et al., 2017).

The research of FD approaches able to improve performance of the system considering both the fault and uncertainty/disturbance effect still is an active area of research (Pan \& Yang, 2017) even though already exist important contributions in the last years (Chen \& Patton, 2012; Ding, 2008; Zhong et al., 2003). In this regard, there are several approaches associated with generating the residual (Chen \& Patton, 1999; Gertler, 1998). So far, one of the most used approach for generating the residual is interval observer-based approach (Puig et al., 2003). Interval observer-based approaches is mainly used to estimate the states from the measurements using either stochastic (e.g., Kalman filters) or deterministic approaches (e.g., Luenberger observers) for modeling the uncertainties. Then, the FD test is done based on generating the residual using the output estimation error (Alamo, Bravo, \& Camacho, 2005; Chen \& Patton, 1999; Combastel, 2015; Puig et al., 2003).

Recent developments in the field of model-based FD have led to a renewed interest in using settheoretical approaches in FD framework. Set-invariance approach is one of the techniques where the invariant sets for the residual can be computed in each healthy or faulty operation of the system (Kofman, 2005; Kofman, Haimovich, \& Seron, 2007; Ocampo-Martinez, De Doná, \& Seron, 2010; Seron, Zhuo, De Doná, \& Martínez, 2008). Consequently, when the system reaches the steady state, the corresponding residual trajectory ultimately converges to one of these invariant sets. As long as both healthy and faulty sets are separated, the FD can be performed (Ocampo-Martinez et al., 2010; Seron et al., 2008).

The Robust Positively Invariant (RPI) set defined is a bounded region in state-space that the system state can be confined in spite of the effect of the bounded system uncertainties (Seron \& De Doná, 2010; Xu, Stoican, Puig, Ocampo-Martinez, \& Olaru, 2013). Furthermore, the minimal Robust Positively Invariant (mRPI) set is a unique and compact RPI set that contained in any closed RPI set (Rakovic, Kerrigan, Kouramas, \& Mayne, 2005; Seron et al., 2008). One major drawback of the set-invariance approach is related to the limitation of computing the finite de- 
scription of the RPI set. In recent years, researchers have investigated a variety of approaches. So far, the proposed approaches can be classified into two main categories: (i) explicit approaches, where the RPI set is computed using the explicit formulation of the set boundary (Kofman et al., 2007), (ii) iterative approaches, where the recursive iteration of the approximation of the system dynamics can be used to reach the RPI set (Artstein \& Raković, 2008; Stoican, Hovd, \& Olaru, 2013). Moreover, the set-invariance approach can be used for checking the separation of healthy and faulty residual sets in steady state.

The minimum detectable fault (MDF) is a typical performance index used to characterize the performance of the FD scheme. The way of computing the MDF depends on the particular modelbased approach and the way that faults and uncertainty are modeled. One possible way to model the fault is based on considering it either as a state or as an unknown input of a dynamic system and the estimation of the fault is done by means of an observer. Alternatively, the fault can be modeled as a uncertain parameter that should be estimated by means of parameter estimation schemes (Shen, Ding, \& Wang, 2013; Tabatabaeipour \& Bak, 2014; Tan, Tao, \& Qi, 2013). Moreover, the MDF is highly affected by the gain of the observer due to its influence in the FD performance. The effect of the observer gain in FD has already been explored in (Meseguer, Puig, Escobet, \& Saludes, 2010).

In (Xu et al., 2013), the relation between the classical interval observer-based approach and the set-invariance approach is discussed. Then, in (Kodakkadan et al., 2017; Pourasghar, Puig, \& Ocampo-Martinez, 2016), the characterization of the minimum magnitude of the fault that can be detected is proposed using both the interval observer-based and set-invariance approaches. However, there has been little discussion about the combination of the mentioned approaches. Then, interval observer-based approach and the set-invariance approach are still known as two different techniques in FD framework. In this regard, this paper is devoted to develop a passive robust FD approach using interval observer-based approach in combination with set-invariance approach. Moreover, another contribution of the paper is to determine the MDF using the proposed approach. System uncertainty is assumed to be unknown but bounded. This fact implies that the interval observerbased approach can deliver no just a single punctual estimation for the output/state but a set that, in this paper, is bounded using zonotopes. On the one hand, the characterization of the MDF is done by using the classical sensitivity analysis. On the other hand, this characterization is carried out by using set-invariance theory. Mathematical expressions of the MDF for different type of sensor and actuator faults are obtained with the goal of connecting both sensitivity and set-based approaches. Contrary to most existing FD design techniques, the sensitivity to both faults and disturbances is evaluated using a set-based approach enclosing all the possible temporal scenarios of faults and disturbances within specified ranges. The combination of these features makes the approach original compared the existing available FD techniques. Finally, the effectiveness of the proposed approach is illustrated using a case study based on a quadruple-tank system.

The structure of the paper is the following: The problem formulation is presented in Section 2 . The observer structure and the FD algorithm are introduced in Section 3. Then, the MDF is characterized in Section 4 dealing with different type of sensor and actuator faults. The case study description and discussion of the obtained results are presented in Section 5. Finally, the general conclusion is drawn in Section 6.

\section{Notation}

Throughout this paper, $\mathbb{R}^{n}$ denotes the set of $n$-dimensional real numbers, $\oplus$ denotes the Minkowski sum. The matrices are written using capital letter, e.g., $A$, the calligraphic notation is used for denoting sets, e.g., $\mathcal{X}$, the transfer functions are highlighted using script font e.g., $\mathscr{H},\|\cdot\|_{s}$ denotes the $s$-norm, $[\underline{x}, \bar{x}]$ is an interval with lower bound $\underline{x}$ and upper bound $\bar{x}$ and the subscript or superscript $i o$ and $i s$ denoted the interval observer-based and set-invariance approaches, respectively. 
In the Appendix, the background on zonotopes required to follow the paper is presented.

\section{Problem formulation}

The state-space form of the system to be monitored in this paper is described by a linear uncertain dynamic model in discrete-time as follows:

$$
\begin{aligned}
x_{k+1} & =A x_{k}+B u_{k}+E_{\omega} \omega_{k}, \\
y_{k} & =C x_{k}+E_{v} v_{k}
\end{aligned}
$$

where $u \in \mathbb{R}^{n_{u}}, y \in \mathbb{R}^{n_{y}}$ and $x \in \mathbb{R}^{n_{x}}$ are the input, the output and the state vectors, respectively. Besides, $A \in \mathbb{R}^{n_{x} \times n_{x}}, B \in \mathbb{R}^{n_{x} \times n_{u}}$ and $C \in \mathbb{R}^{n_{y} \times n_{x}}$ are the state-space matrices, while $\omega \in \mathbb{R}^{n_{x}}$ and $v \in \mathbb{R}^{n_{y}}$ are measurement disturbance and process noise vectors, respectively. Moreover, $E_{\omega}$ and $E_{v}$ are the empty matrices with appropriate dimensions while $k \in \mathbb{N}$ indicates the discrete time.

The measurement noise and process disturbances are assumed to be unknown but bounded, i.e.,

$$
\begin{aligned}
\mathcal{W} & =\left\{\omega_{k} \in \mathbb{R}^{n_{x}}:\left|\omega_{k}-c_{\omega}\right| \leq \bar{\omega}, c_{\omega} \in \mathbb{R}^{n_{x}}, \bar{\omega} \in \mathbb{R}^{n_{x}}\right\}, \\
\mathcal{V} & =\left\{v_{k} \in \mathbb{R}^{n_{y}}:\left|v_{k}-c_{v}\right| \leq \bar{v}, c_{v} \in \mathbb{R}^{n_{y}}, \bar{v} \in \mathbb{R}^{n_{y}}\right\},
\end{aligned}
$$

where $c_{\omega}, \bar{\omega}, c_{v}$ and $\bar{v}$ are constant vectors. Furthermore, (2) can be rewritten as a zonotopic representation of the set as

$$
\begin{aligned}
\mathcal{W} & =\left\langle c_{\omega}, R_{\omega}\right\rangle, \\
\mathcal{V} & =\left\langle c_{v}, R_{v}\right\rangle,
\end{aligned}
$$

where $c_{\omega}$ and $c_{v}$ denote the centers of the disturbance and noise bouding zonotopes, respectively, with their generator matrices $R_{\omega} \in \mathbb{R}^{n_{x} \times n_{x}}$ and $R_{v} \in \mathbb{R}^{n_{y} \times n_{y}}$, respectively.

Henceforth, the index $k+1$ will be replaced by + for the sake of simplified notation. In this regard, the dynamical model in (1) can be rewritten as

$$
\begin{aligned}
x_{+} & =A x+B u+E_{\omega} \omega \\
y & =C x+E_{v} v .
\end{aligned}
$$

Assumption 2.1: The pair $\{A, C\}$ is detectable.

Assumption 2.2: Disturbance and noise bounds represented in (3) are assumed to be bounded by a unitary hypercube zonotopes centered at the origin, i.e., $\forall k \geq 0, \omega=[-1,1]=\left\langle 0, I_{n_{\omega}}\right\rangle$ and $v=[-1,1]=\left\langle 0, I_{n_{v}}\right\rangle$ where $I_{n_{\omega}} \in \mathbb{R}^{n_{\omega} \times n_{\omega}}$ and $I_{n_{v}} \in \mathbb{R}^{n_{v} \times n_{v}}$ denote the identity matrices.

\section{FD test}

\subsection{Interval observer-based FD test}

FD test in the interval observer-based approach consists in testing whether the system measurements are consistent with the behavior described by the system model in healthy operation. The existence of the fault is demonstrated in the case that measurements are inconsistent with the behavior of the model. Monitoring the dynamical model (4) can be done by designing a Luenberger 
observer of the form

$$
\hat{x}_{+}=A \hat{x}+B u+K(y-\hat{y}),
$$

where $\hat{x}$ is the state estimation. Moreover, the observer gain $K$ should be computed such that $(A-K C)$ is a Schur matrix.

Assumption 3.1: The initial state $x_{0}$ belongs to the zonotopic set $\mathcal{X}_{0}=\left\langle c_{0}, R_{0}\right\rangle$, where $c_{0} \in \mathbb{R}^{n_{x}}$ denotes the center and $R_{0} \in \mathbb{R}^{n_{x} \times n_{R_{0}}}$ is a non-empty matrix containing the generators matrix of the initial zonotope $\mathcal{X}_{0}$.

Therefore, according to Xu, Puig, Ocampo-Martinez, Olaru, \& Stoican (2015), the resulting interval observation of the dynamical model (4) can be defined by using Proposition 3.1.

Proposition 3.1: (Zonotopic-observer structure) Considering the observer (5) and Assumptions 2.2 and 3.1, the center $c$ and the shape matrix $R$ of the state-bounding observer can be recursively defined as

$$
\begin{aligned}
c_{+} & =(A-K C) c+B u+K y, \\
R_{+} & =\left[\begin{array}{lll}
(A-K C) \bar{R} & E_{\omega} & -K E_{v}
\end{array}\right],
\end{aligned}
$$

where $\bar{R}=\downarrow_{q}\{R\}$. Moreover, the state inclusion property $x \in\langle c, R\rangle$ holds for all $k \geq 0$ (see Properties 3 and 4 in the Appendix).

Proof. Assume $x \in\langle c, R\rangle, \omega \in\left\langle 0, I_{n_{\omega}}\right\rangle$ and $v \in\left\langle 0, I_{n_{v}}\right\rangle$ where the inclusion property (see Property 2 in the Appendix) is preserved by using the reduction operator, which means $x \in\langle c, \bar{R}\rangle$. Thus, the set-based interval observation can be written using (5) as

$$
x_{+} \in\left\langle c_{+}, R_{+}\right\rangle=\langle(A-K C) c,(A-K C) \bar{R}\rangle \oplus\left\langle B_{u} u, 0\right\rangle \oplus\left\langle 0, E_{\omega}\right\rangle \oplus\langle K y, 0\rangle \oplus\left\langle 0,-K E_{v}\right\rangle .
$$

Thus, based on Definition 2 and Property 1, $c_{+}$and $R_{+}$in (7) can be derived as in (6).

Likewise, the output can be predicted using Proposition 3.2.

Proposition 3.2: Considering the dynamical model (4) and Proposition 3.1, $c_{y}$ and $R_{y}$ defining the output-bounding zonotopic set $\left\langle c_{y}, R_{y}\right\rangle$ can be computed as

$$
\begin{aligned}
c_{y} & =C c, \\
R_{y} & =\left[\begin{array}{ll}
C \bar{R} & E_{v}
\end{array}\right] .
\end{aligned}
$$

Proof. Assume $x \in\langle c, R\rangle$ and $v \in\left\langle 0, I_{n_{v}}\right\rangle$ for all $k \geq 0$, where the inclusion is preserved by using the reduction operator, which means $x \in\langle c, \bar{R}\rangle$. Thus, (4b) can be written as

$$
y \in\left\langle c_{y}, R_{y}\right\rangle=\langle C c, C \bar{R}\rangle \oplus\left\langle 0, E_{v}\right\rangle .
$$

Therefore, based on Definition 2 and Property 1, $c_{y}$ and $R_{y}$ in (9) can be expressed as (8). This gives the proof of Proposition 3.2.

Now, the residual can be generated using the Proposition 3.3.

Proposition 3.3: (Residual generation using interval observer-based approach) Considering Proposition 3.2 and the measurement equation in (4b), $c_{r^{i o}}$ and $R_{r^{i o}}$ of the residual zonotopic 
set are generated as

$$
\begin{aligned}
c_{r^{i o}} & =y-C c, \\
R_{r^{i o}} & =\left[\begin{array}{ll}
-C \bar{R} & -E_{v}
\end{array}\right],
\end{aligned}
$$

where the subscript $r^{i o}$ denotes that obtained residual is generated using the interval observer-based approach.

Proof. The output equation (4b) can be written as

$$
0=y-C x-E_{v} v .
$$

Now, considering $x \in\langle c, R\rangle$ and $v \in\left\langle 0, I_{n_{v}}\right\rangle$, (11) becomes

$$
0 \in\langle y, 0\rangle \oplus\langle-C c,-C R\rangle \oplus\left\langle 0,-E_{v}\right\rangle,
$$

where $c$ is known using observer (6). Thus, considering Definition 2 and Property $1, c_{r^{i o}}$ and $R_{r^{i o}}$ in (12) can be expressed as in (10). This gives the proof of Proposition 3.3.

Hence, the FD test can be done by checking the satisfaction of $0 \notin\left\langle c_{r^{i o}}, R_{r^{i o}}\right\rangle$. A computationally efficient and widely used way to implement the detection test without increasing the false alarm rate consists in testing whether or not 0 belongs to an aligned box enclosing the zonotope $\left\langle c_{r^{i o}}, R_{r^{i o}}\right\rangle$, i.e.,

$$
0 \notin\left\langle c_{r^{i o}}, b\left(R_{r^{i o}}\right)\right\rangle,
$$

where $\left\langle c_{r^{i o}}, b\left(R_{r^{i o}}\right)\right\rangle$ is enclosed by an aligned box denoted by $b\left(R_{r^{i o}}\right)$.

Algorithm 1 summarizes the FD test procedure using interval observer-based apprach.

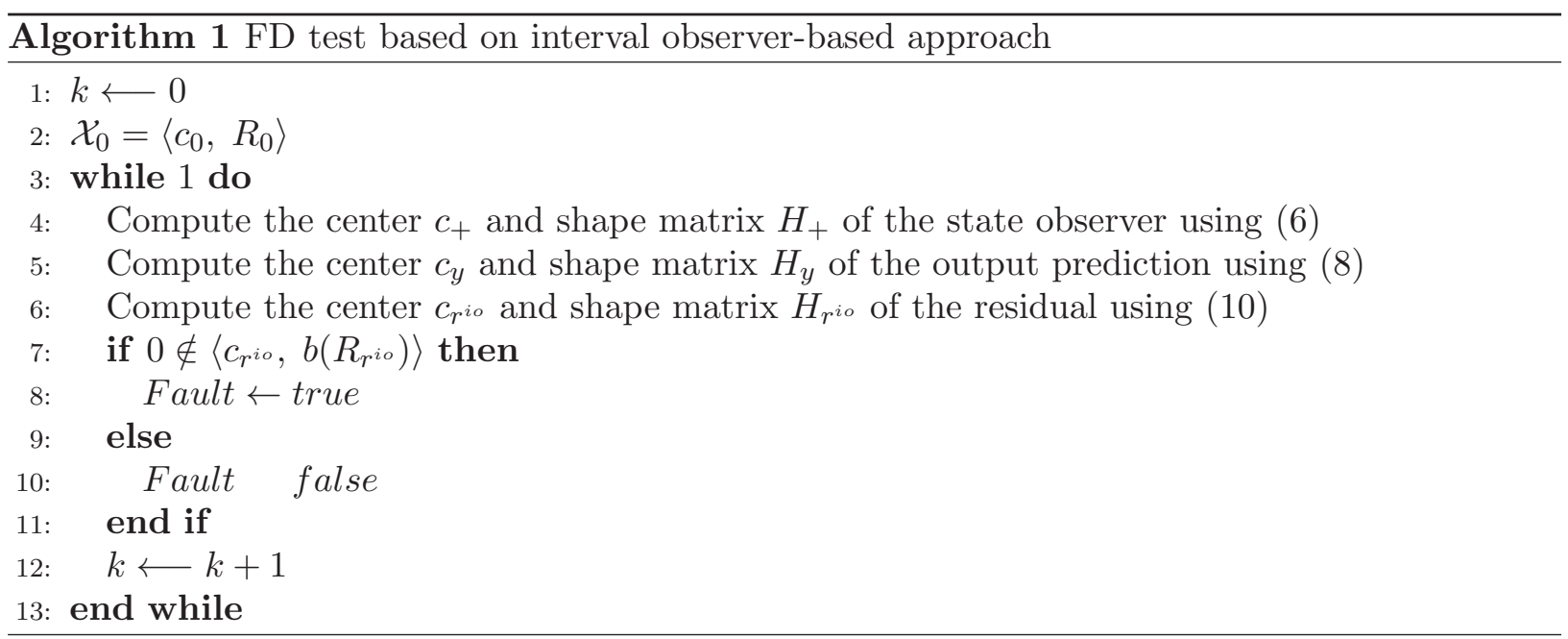

The FD test in Algorithm 1 is based on checking if 0 is inside the residual zonotopic set. This set is propagated online to detect the existence of the fault. Alternatively, one possible way for detecting the fault is to make use of the set-invariance approach which will be discussed in next section. 


\subsection{Set-invariance-based FD test}

The set-invariance-based FD test also relies on a Luenberger observer (5) for monitoring the dynamical model (4). In this regard, the dynamics of the state estimation error, denoted by $\tilde{x}$, are stated as

$$
\tilde{x}_{+}=(A-K C) \tilde{x}+E_{\omega} \omega-K E_{v} v,
$$

where $\tilde{x}=x-\hat{x}$ and (14) is stable since $(A-K C)$ is a Schur matrix. Furthermore, the residual in the case of the set-invariance approach, namely $r^{\text {is }}$, can be computed by considering the measured output vector $y$ as

$$
r^{\text {is }}=y-C \hat{x}=C \tilde{x}+E_{v} v .
$$

Now, according to Kolmanovsky \& Gilbert (1998) and assuming bounded disturbances and noises (Assumption 2.2), an invariant set denoted by $\tilde{\mathcal{X}}$ can be constructed, which includes the state estimation error $\tilde{x}$. It means, $\tilde{x}$ evaluation remains inside $\tilde{\mathcal{X}}$ if it is started from inside. Otherwise, when $\tilde{x}$ evaluation is started from outside $\tilde{\mathcal{X}}$, it can enter this set after a finite time. Generally speaking, $\tilde{\mathcal{X}}$ is known as robust positive invariant (RPI) set if for any $\tilde{x} \in \tilde{\mathcal{X}}, \omega \in \mathcal{W}$ and $v \in \mathcal{V}$, then, $\tilde{x}_{+} \in \tilde{\mathcal{X}}, \forall k \geq 0$ holds. Therefore,

$$
(A-K C) \tilde{x}+E_{\omega} \omega-K E_{v} v \in \tilde{\mathcal{X}}, \quad \forall \omega \in \mathcal{W} \text { and } v \in \mathcal{V}
$$

also holds.

While a variety of methods has been developed to construct the invariant set $\tilde{\mathcal{X}}$ according to Kofman (2005), this paper uses the ultimate bounds method reported by Kofman et al. (2007). In this regard, the Jordan Canonical form of $(A-K C)$ is computed by

$$
J=V(A-K C) V^{-1},
$$

where $J$ is a diagonal matrix corresponding to the Jordan-normal form of $(A-K C)$ and $V$ is a non-singular transformation matrix. Therefore, $\tilde{x}$ and its dynamics in (14) ultimately converge within the RPI set $\tilde{\mathcal{X}}$ defined as

$$
\tilde{\mathcal{X}}=\left\{\tilde{x} \in \mathbb{R}^{n_{x}}:\left|V^{-1} \tilde{x}\right| \leq(I-|J|)^{-1}\left|V^{-1}\right| \bar{\omega}+\varepsilon\right\},
$$

where $\varepsilon$ can be any arbitrary small vector with strictly positive components.

So far, the paper is mainly focused on using the zonotopic representation of sets. But, the obtained set in (18) is a polyhedral RPI set of the state estimation error. In this regard, since the set in (18) is symmetric around the origin and considering the zonotopic representation of sets, Proposition 3.4 implies that $\tilde{\mathcal{X}}$ can also be represented using a zonotope.

Proposition 3.4: (Zonotopic representation of $\tilde{\mathcal{X}}$ ) Considering the steady-state operation of the system and Proposition 3.1, the polyhedral RPI set of $\tilde{x}$ in (18) can be obtained using Definition 3 in the Appendix in its zonotopic representation as

$$
\begin{aligned}
c_{\tilde{x}_{\infty}} & =0, \\
\left\|R_{\tilde{x}_{\infty_{i}}}\right\|_{1} & =\left\|R_{\infty_{i}}\right\|_{1},
\end{aligned}
$$

where $c_{\tilde{x}_{\infty}}$ and $R_{\tilde{x}_{\infty_{i}}}$ are the center and the shape (generator) matrix of the zonotopic set $\tilde{\mathcal{X}}$, respectively. Moreover, $i$ represent the $i$-row of the matrix $R_{\tilde{x}}$ and $R$ in (6) when $k$ tends to infinity. 
Proof. Given the state estimation error dynamics in (14) and assuming the initial state estimation error $\tilde{x}_{0}$ belongs to the zonotopic set $\tilde{\mathcal{X}}_{0}=\left\langle c_{\tilde{x}_{0}}, R_{\tilde{x}_{0}}\right\rangle$, defined as an RPI set, since $\tilde{x} \in\left\langle c_{\tilde{x}}, R_{\tilde{x}}\right\rangle$, $\omega \in\left\langle 0, I_{n_{\omega}}\right\rangle$ and $v \in\left\langle 0, I_{n_{v}}\right\rangle$ for all $k \geq 0$, the set

$$
\tilde{x}_{j+1} \in\left\langle c_{\tilde{x}_{j+1}}, R_{\tilde{x}_{j+1}}\right\rangle=\left\langle(A-K C) c_{\tilde{x}_{j}},(A-K C) R_{\tilde{x}_{j}}\right\rangle \oplus\left\langle 0, E_{\omega}\right\rangle \oplus\left\langle 0,-K E_{v}\right\rangle
$$

converges to the mRPI set (i.e., an RPI set contained in any closed RPI set) when $k$ tends to infinity, where $j \in \mathbb{N}$ represents the $j$-th element of the set. Thus, the center and the shape matrix of the set in (20) can be unfolded as

$$
\begin{aligned}
c_{\tilde{x}_{j+1}} & =(A-K C) c_{\tilde{x}_{j}}, \\
R_{\tilde{x}_{j+1}} & =\left[\begin{array}{lll}
(A-K C) R_{\tilde{x}_{j}} & E_{\omega} & -K E_{v}
\end{array}\right] .
\end{aligned}
$$

Furthermore, $\tilde{x}$ converges to $\tilde{\mathcal{X}}$ in steady state with the iterative propagation of (21) by starting from the initial set $\tilde{\mathcal{X}}_{0}=\left\langle c_{\tilde{x}_{0}}, R_{\tilde{x}_{0}}\right\rangle$ that belongs to the RPI set. Hence, in the case that $j$ tends to infinity (in order to reach steady state), then the following conditions can be written:

$$
\begin{aligned}
c_{\tilde{x}_{j+1}} & =c_{\tilde{x}_{j}}, \\
\left\|R_{\tilde{x}_{j+1}}\right\|_{1} & =\left\|R_{\tilde{x}_{j}}\right\|_{1} .
\end{aligned}
$$

Therefore, the same formulations as (19) for the center and the shape matrix of $\tilde{\mathcal{X}}$ can be obtained by rewriting (21) based on the conditions in (22). This gives the proof of Proposition 3.4.

Consequently, the corresponding residual set can be computed using Proposition 3.5.

Proposition 3.5: Considering (15) and Proposition 3.4, $c_{r^{i s}}$ and $R_{r^{i s}}$ of the residual using setinvariance approach are generated as

$$
\begin{aligned}
c_{r_{\infty}^{i s}} & =C c_{\tilde{x}_{\infty}}=0, \\
R_{r_{\infty}^{i s}} & =\left[\begin{array}{ll}
C R_{\tilde{x}_{\infty}} & E_{v}
\end{array}\right],
\end{aligned}
$$

where the subscript $r^{i s}$ denotes the residual set is generated based on set-invariance approach which is invariant when $k$ tends to infinity.

Proof. Considering $\tilde{\mathcal{X}}_{j}=\left\langle c_{\tilde{x}_{j}}, R_{\tilde{x}_{j}}\right\rangle$ based on Proposition 3.4 and initializing the system state by starting the iterative propagation from the zonotopic RPI set i.e., $\tilde{\mathcal{X}}_{0}=\left\langle c_{\tilde{x}_{0}}, R_{\tilde{x}_{0}}\right\rangle$, and also assuming $v \in\left\langle 0, I_{n_{v}}\right\rangle$, the zonotopic residual set can be obtained using (15) as

$$
\left\langle c_{r_{j}^{i s}}, R_{r_{j}^{i s}}\right\rangle=\left\langle C c_{\tilde{x}_{j}}, C R_{\tilde{x}_{j}}\right\rangle \oplus\left\langle 0, E_{v}\right\rangle
$$

Furthermore, it can be considered that whenever $\tilde{x}_{j}$ is inside of $\left\langle c_{\tilde{x}_{j}}, R_{\tilde{x}_{j}}\right\rangle, r_{j}^{i s}$ remains inside of $\left\langle c_{r_{j}^{i s}}, R_{r_{j}^{i s}}\right\rangle$. Now, using the same way as the proof of Proposition 3.4, by considering the steady state operation, the center and the shape matrix of the residual in this case can be obtained as in (23). This gives the proof of Proposition 3.5.

Finally, considering the residual that is obtained in Proposition 3.5 as a healthy residual set that is generated based on the nominal model, i.e., considering only the effect of the uncertainties (noises and disturbances), the FD test with the set-invariance approach is done by comparing the residual that is computed on-line at each time instant, denoted by $r$, with the RPI residual set 
that is computed off-line in steady state. The fault is detected if

$$
r \notin\left\langle c_{r_{\infty}^{i s}}, R_{r_{\infty_{i}}^{i s}}\right\rangle .
$$

Otherwise, the system remains healthy.

Remark 1: From the mathematical point of view, in order to check whether (25) is satisfied or not, the Gilbert-Johnson-Keerthi (GJK) algorithm as proposed in (Lalami \& Combastel, 2006) can be used.

\subsection{Integrated FD test}

So far, both interval observer-based and set-invariance approaches are considered as reliable settheoretical FD tests that can be used depending on the purpose of the investigation. In general, the FD test is provided based on checking the consistency of the residual $r$ in real time, which is computed on-line, with the healthy residual zonotope that can be computed based on either interval observer-based or set-invariance approaches. Then, any inconsistencies can be understood as the occurrence of the fault. Therefore, Proposition 3.6 can be used in order to combine the FD principles based on both interval-observer and set-invariance approaches.

Proposition 3.6: Considering the steady state and the general Luenberger observer structure in (5), in healthy operation of the system

$$
\left\langle c_{r_{\infty}^{i o}}, R_{r_{\infty}^{i o}}\right\rangle=\left\langle c_{r_{\infty}^{i s}}, R_{r_{\infty}^{i s}}\right\rangle .
$$

Then, the FD test is the same using both interval observer-based and set-invariance approaches and can be done by checking whether

$$
r \in\left\langle c_{r_{\infty}^{h}}, R_{r_{\infty}^{h}}\right\rangle
$$

where $c_{r^{h}}$ and $R_{r^{h}}$ are the center and the shape (generator) matrix of the healthy residual zonotope denoted by $r^{h}$, respectively (i.e., $r^{h}=\left\langle c_{r^{h}}, R_{r^{h}}\right\rangle$ ). Moreover, $c_{r^{h}}$ and $R_{r^{h}}$ can be computed using set-invariance approach or interval observer-based approach when $k$ tends to infinity.

Proof. The residual in the case of interval observer-based approach can be written using the state estimation error as

$$
r^{i o}=C \tilde{x}^{i o}+E_{v} v
$$

where $\tilde{x}^{i o}$ is the state estimation error using using the observer structure in Proposition 3.1. Moreover, the following dynamics of the state estimation error using interval observer-based approach can be written using the same Luenberger observer structure as in (5):

$$
\tilde{x}_{+}^{i o}=(A-K C) \tilde{x}^{i o}+E_{\omega} \omega-K E_{v} v
$$

where, generally speaking, $\tilde{x}^{i o}$ is different from $\tilde{x}$ in (14) that indicates the state estimation error based on set-invariance approach. But, based on Xu et al. (2013), considering the steady-state operation of the system, the size of the residual set $\left\langle c_{r_{\infty}^{i o}}, R_{r_{\infty}^{i o}}\right\rangle$ obtained using the interval observerbased approach converges towards the smallest residual set (healthy set) computed by the setinvariance approach $\left\langle c_{r_{\infty}^{i s}}, R_{r_{\infty}^{i s}}\right\rangle$. Therefore, the same residual zonotopes can be predicted based on both the interval observer-based and set-invariance approaches, if $k$ tends to infinity (steady 
state). Then, since having the same set that introduces the healthy residual, the same FD principle can be obtained using both approaches.

Then, considering Proposition 3.6, residual (28) or (15) can be used to compute the healthy residual zonotope in steady state. Note that for the sake of simplified notation, $r^{h}$ is used instead of $r^{i o}$ or $r^{i s}$ for denoting the healthy residual since they are equal in steady state dealing with the faultless scenario.

In this regard, the input-output form of the output $y$ in (4) can be written using the shift operator $q^{-1}$ as

$$
y=\mathscr{H}_{u}\left(q^{-1}\right) u+\mathscr{H}_{\omega}\left(q^{-1}\right) \omega+\mathscr{H}_{v}\left(q^{-1}\right) v
$$

with

$$
\begin{aligned}
\mathscr{H}_{u}\left(q^{-1}\right) & =C \xi_{1}\left(q^{-1}\right) B, \\
\mathscr{H}_{\omega}\left(q^{-1}\right) & =C \xi_{1}\left(q^{-1}\right) E_{\omega}, \\
\mathscr{H}_{v}\left(q^{-1}\right) & =E_{v},
\end{aligned}
$$

where $\xi_{1}\left(q^{-1}\right)=(q I-A)^{-1}$.

On the other hand, the input-output form of the output prediction $\hat{y}$ can be expressed using observer (5) as

$$
\hat{y}=\mathscr{T}_{u}\left(q^{-1}\right) u+\mathscr{T}_{y}\left(q^{-1}\right) y,
$$

with

$$
\begin{aligned}
& \mathscr{T}_{u}\left(q^{-1}\right)=C \xi_{2}\left(q^{-1}\right) B, \\
& \mathscr{T}_{y}\left(q^{-1}\right)=C \xi_{2}\left(q^{-1}\right) K,
\end{aligned}
$$

where $\xi_{2}\left(q^{-1}\right)=(q I-(A-K C))^{-1}$.

Now, using (30) and (32), the input-output form of the healthy residual can be expressed using the shift operator $q^{-1}$ as

$$
r^{h}=\mathscr{G}_{u}\left(q^{-1}\right) u+\mathscr{G}_{\omega}\left(q^{-1}\right) \omega+\mathscr{G}_{v}\left(q^{-1}\right) v,
$$

with

$$
\begin{aligned}
& \mathscr{G}_{u}\left(q^{-1}\right)=\left(I-\mathscr{T}_{y}\left(q^{-1}\right)\right) \mathscr{H}_{u}\left(q^{-1}\right)-\mathscr{T}_{u}\left(q^{-1}\right), \\
& \mathscr{G}_{\omega}\left(q^{-1}\right)=\left(I-\mathscr{T}_{y}\left(q^{-1}\right)\right) \mathscr{H}_{\omega}\left(q^{-1}\right), \\
& \mathscr{G}_{v}\left(q^{-1}\right)=\left(I-\mathscr{T}_{y}\left(q^{-1}\right)\right) \mathscr{H}_{v}\left(q^{-1}\right) .
\end{aligned}
$$

As it can be seen from (34), the obtained residual is only affected by the additive uncertainties (disturbance and noise). Furthermore, $\mathscr{G}_{\omega}$ and $\mathscr{G}_{v}$ indicate the sensitivity of the residual with respect to the disturbance and noise, respectively.

Thus, the obtained healthy residual in (34) can be used for the FD test in (27) following Proposition 3.7 .

Proposition 3.7: Considering the input-output form of the residual in (34), the FD test in (27) 
can be also done by checking the satisfaction of

$$
r \in \mathscr{G}_{u_{i}}(1) u_{\infty} \pm\left\|\mathscr{G}_{\omega_{i}}(1)+\mathscr{G}_{v_{i}}(1)\right\|_{1},
$$

where the index $i$ indicates the $i^{\text {th }}$ line of the transfer function $\mathscr{G}$. In case that (36) is not satisfied a fault is detected.

Proof. Since $u \in\langle u, 0\rangle, \omega \in\left\langle 0, I_{n_{\omega}}\right\rangle$ and $v \in\left\langle 0, I_{n_{v}}\right\rangle$ for all $k \geq 0$, the healthy residual set in steady state can be written as

$$
r_{\infty}^{h} \in\left\langle c_{r_{\infty}^{h}}, R_{r_{\infty}^{h}}\right\rangle=\left\langle\mathscr{G}_{u}(1) u_{\infty}, 0\right\rangle \oplus\left\langle 0, \mathscr{G}_{\omega}(1)\right\rangle \oplus\left\langle 0, \mathscr{G}_{v}(1)\right\rangle .
$$

Note that the steady state expression of (34) can be obtained by stating $q=1$. Then, (37) can also be expressed as a zonotopic representation as

$$
\begin{aligned}
c_{r_{\infty}^{h}} & =\mathscr{G}_{u}(1) u_{\infty}, \\
R_{r_{\infty}^{h}} & =\left[\begin{array}{ll}
\mathscr{G}_{\omega}(1) & \mathscr{G}_{v}(1)
\end{array}\right] .
\end{aligned}
$$

Now, by considering both Definition 3 in the Appendix and (38), upper and lower bounds of the residual zonotope, which are respectively denoted by $\bar{r}^{h}$ and $\underline{r}^{h}$, can be computed as

$$
\begin{aligned}
\bar{r}_{\infty}^{h} & =\mathscr{G}_{u_{i}}(1) u_{\infty}+\left\|\mathscr{G}_{\omega_{i}}(1)+\mathscr{G}_{v_{i}}(1)\right\|_{1}, \\
\underline{r}_{\infty}^{h} & =\mathscr{G}_{u_{i}}(1) u_{\infty}-\left\|\mathscr{G}_{\omega_{i}}(1)+\mathscr{G}_{v_{i}}(1)\right\|_{1} .
\end{aligned}
$$

Thus, considering (39) as upper and lower bounds, the fault can be detected if $r \notin\left[\underline{r}_{\infty}^{h}, \bar{r}_{\infty}^{h}\right]$.

Hence, the fault can be detected by generating the residual in real time and comparing it with the residual set that was computed off-line using Proposition 3.7. A deeper discussion about the computation of the minimum magnitude of the fault that can be detected using the approach presented in this section will be done in Section 4.

\section{Characterization of the MDF using sensitivity analysis integrated with a set-invariance approach}

Returning to the main issue posted at the beginning of this paper, the characterization of the MDF is the main aim addressed in this section. In this paper, MDF is defined as the minimum magnitude of the fault that can be detected. Furthermore, faults are generally classified into two types: sensor and actuator faults. Figure 1 shows the schematic graphical interpretation of the different actuator and sensors faults.

Moreover, the combination of classical sensitivity analysis and the set-invariance approach (called integrated approach in Section 3) is employed to deal with the computation of the MDF.

Assumption 4.1: The fault $f$ is considered as an additive (abrupt) fault that is bounded by a unit hypercube expressed as the centered zonotope in all the cases, i.e., $\forall k \geq 0, f=[-1,1]=\left\langle 0, I_{n_{f}}\right\rangle$. 


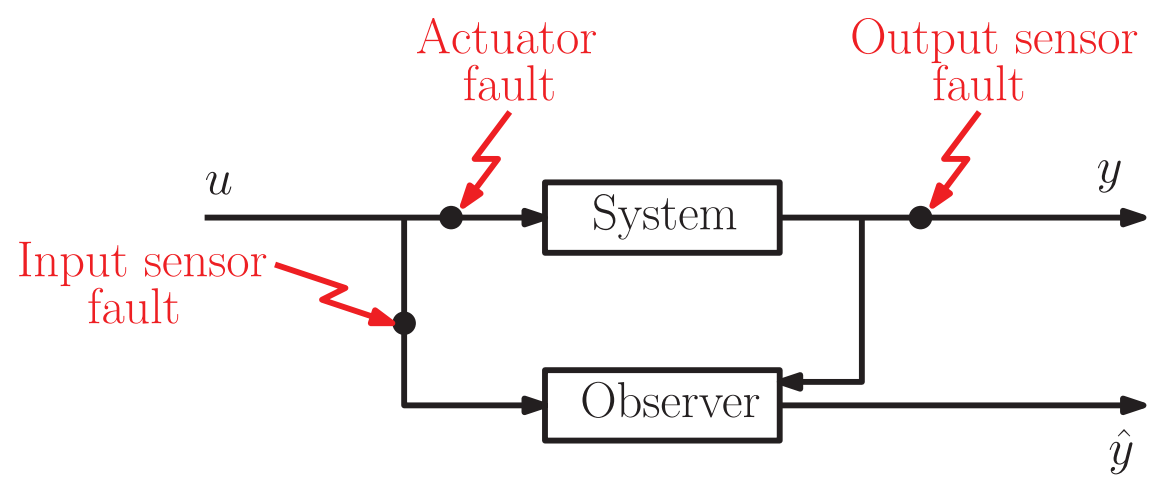

Figure 1.: Graphical interpretation of the different actuator and sensors faults.

\subsection{Minimum detectable output sensor fault}

The following dynamical model is considered to deal with the output sensor fault:

$$
\begin{aligned}
x_{+} & =A x+B u+E_{\omega} \omega, \\
y & =C x+E_{v} v+F_{y} f_{y},
\end{aligned}
$$

where $f_{y}$ denotes the output sensor fault and $F_{y}$ denotes the associated matrix by the given fault with suitable dimensions as

$$
F_{y} \in \mathbb{R}^{n_{y} \times n_{y}} .
$$

The dynamic system in (40) can be written in input-output form using the shift operator $q^{-1}$ as

$$
y=\mathscr{H}_{u}\left(q^{-1}\right) u+\mathscr{H}_{\omega}\left(q^{-1}\right) \omega+\mathscr{H}_{v}\left(q^{-1}\right) v+\mathscr{H}_{f_{y}}\left(q^{-1}\right) f_{y}
$$

where

$$
\mathscr{H}_{f_{y}}\left(q^{-1}\right)=F_{y} .
$$

Furthermore, using the Luenberger observer (5), the output prediction can be expressed in inputoutput form as in (32). Therefore, the input-output form of the residual in the case of output sensor fault can be expressed as

$$
r=\mathscr{G}_{u}\left(q^{-1}\right) u+\mathscr{G}_{\omega}\left(q^{-1}\right) \omega+\mathscr{G}_{v}\left(q^{-1}\right) v+\mathscr{G}_{f_{y}}\left(q^{-1}\right) f_{y},
$$

with

$$
\mathscr{G}_{f_{y}}\left(q^{-1}\right)=\left(I-\mathscr{T}_{y}\left(q^{-1}\right)\right) \mathscr{H}_{f_{y}}\left(q^{-1}\right) .
$$

Therefore, (43) encompasses the effect of uncertainties (disturbance and noise) and also the effect of the fault. Furthermore, $\mathscr{G}_{\omega}\left(q^{-1}\right), \mathscr{G}_{v}\left(q^{-1}\right)$ and $\mathscr{G}_{f_{y}}\left(q^{-1}\right)$ denote the sensitivity of the residual with respect to the disturbance, noise and output sensor fault, respectively.

Now, the minimum detectable output sensor fault can be computed following the Theorem 4.1.

Theorem 4.1: (Minimum detectable output sensor fault) Considering the faulty dynamical model (40) and the observer structure (5), the minimum magnitude of the output sensor fault can be 
characterized as

$$
\begin{aligned}
\bar{f}_{y_{j, \infty}} & =\max \bar{f}_{y_{j i, \infty}}, & \bar{f}_{y_{j i, \infty}} & =+2 \frac{\left\|\left(I-C_{i} \xi_{2_{i}}(1) K_{i}\right)\left(C_{i} \xi_{1}(1) E_{\omega_{i}}+E_{v_{i}}\right)\right\|_{1}}{\left\|\left(I-C_{i j} \xi_{2_{i j}}(1) K_{i j}\right) F_{y_{i j}}\right\|_{1}}, \\
\underline{f}_{y_{j, \infty}} & =\min \underline{f}_{y_{j i, \infty}}, & \underline{f}_{y_{j i, \infty}} & =-2 \frac{\left\|\left(I-C_{i} \xi_{2_{i}}(1) K_{i}\right)\left(C_{i} \xi_{1}(1) E_{\omega_{i}}+E_{v_{i}}\right)\right\|_{1}}{\left\|\left(I-C_{i j} \xi_{2_{i j}}(1) K_{i j}\right) F_{y_{i j}}\right\|_{1}}
\end{aligned}
$$

where $\bar{f}_{y_{j, \infty}}$ and $\underline{f}_{y_{j, \infty}}$ are upper and lower bounds of the MDF in the case of output sensor fault, respectively. Additionally, the indices $i$ and $j$ indicate the $i^{\text {th }}$ line and $j^{\text {th }}$ column of the transfer functions, respectively. Moreover, the value 2 is included to consider the worst-case scenario, i.e., the residual is considered to be in the extreme value (it is located at either the lower or the upper bound of the considered threshold).

Proof. Given a residual in (43), considering the faulty residual in the case of an output sensor fault, and following the FD test presented in Proposition 3.6, it can be written that in faulty scenario (i.e., $f_{y}\left(q^{-1}\right) \neq 0 \Rightarrow f_{y_{\infty}} \neq 0$ ), the following condition should be satisfied in steady state:

$$
r_{\infty} \notin \mathscr{G}_{u_{i}}(1) u_{\infty} \pm\left\|\mathscr{G}_{\omega_{i}}(1)+\mathscr{G}_{v_{i}}(1)\right\|_{1}
$$

where $r_{\infty}$ corresponds to the residual that is obtained at $k=\infty$ using (43). Moreover, since $u \in\langle u, 0\rangle, \omega \in\left\langle 0, I_{n_{\omega}}\right\rangle, v \in\left\langle 0, I_{n_{v}}\right\rangle$ and considering Assumption 4.1, it can be written in steady state $\forall i, j$ that

$$
\mathscr{G}_{f_{y_{i j}}}(1) f_{y_{j, \infty}} \notin 0 \pm 2\left\|\mathscr{G}_{\omega_{i}}(1)+\mathscr{G}_{v_{i}}(1)\right\|_{1} .
$$

Therefore, the MDF in the case of an output sensor fault can be calculated by simplifying (47) as

$$
f_{y_{j, \infty}} \notin 0 \pm 2 \frac{\left\|\mathscr{G}_{\omega_{i}}(1)+\mathscr{G}_{v_{i}}(1)\right\|_{1}}{\left\|\mathscr{G}_{f_{y_{i j}}}(1)\right\|_{1}} .
$$

Finally, the MDF can be characterized as it is formulated in (45) by the substitution of (35b), (35c) and (44) into (48) for the sensitivity with respect to $\omega, v$ and $f_{y}$, respectively.

\subsection{Minimum detectable input sensor fault}

The sensor fault is divided into the output and input sensor faults. The main distinction considered in this paper between output and input sensor faults is related to the location of occurrence of the fault. If the occurrence of the fault is located at the output measurement of the dynamical model, it can be known as a output sensor fault (see (40)). Furthermore, if the input of the observer is affected by the fault, it is known as input sensor fault in this paper. Hence, the faulty observer model (5) including the fault is considered as

$$
\hat{x}_{+}=A \hat{x}+B\left(u+F_{u} f_{u}\right)+K(y-\hat{y}),
$$

where $f_{u}$ indicates the input sensor fault and $F_{u}$ denotes the associated matrix by the given fault with suitable dimensions as

$$
F_{u} \in \mathbb{R}^{n_{u} \times n_{u}} .
$$


Thus, the input-output form of the dynamical model (4) can be written using the shift operator $q^{-1}$ as

$$
y=\mathscr{H}_{u}\left(q^{-1}\right) u+\mathscr{H}_{\omega}\left(q^{-1}\right) \omega+\mathscr{H}_{v}\left(q^{-1}\right) v .
$$

Moreover, the observer (49) affected by the input sensor fault can be expressed as

$$
\hat{y}=\mathscr{T}_{u}\left(q^{-1}\right) u+\mathscr{T}_{y}\left(q^{-1}\right) y+\mathscr{T}_{f_{u}}\left(q^{-1}\right) f_{u},
$$

where

$$
\mathscr{T}_{f_{u}}\left(q^{-1}\right)=C \xi_{2}\left(q^{-1}\right) B F_{u} .
$$

Therefore, using (50) and (51), the input-output form of the residual in the case of an input sensor fault is expressed as

$$
r=\mathscr{G}_{u}\left(q^{-1}\right) u+\mathscr{G}_{\omega}\left(q^{-1}\right) \omega+\mathscr{G}_{v}\left(q^{-1}\right) v+\mathscr{G}_{f_{u}}\left(q^{-1}\right) f_{u}
$$

with

$$
\mathscr{G}_{f_{u}}\left(q^{-1}\right)=-\mathscr{T}_{f_{u}}\left(q^{-1}\right) .
$$

Furthermore, (53) allows to decouple the residual obtained with respect to the effect of the uncertainties (additive disturbance and noise) and the considered type of fault. In (53), $\mathscr{G}_{\omega}\left(q^{-1}\right)$ and $\mathscr{G}_{v}\left(q^{-1}\right)$ indicate the sensitivity of the residual with respect to the disturbance $\omega$ and the measurement noise $v$, respectively. Moreover, the sensitivity of the obtained residual with respect to the input sensor fault is indicated by $\mathscr{G}_{f_{u}}\left(q^{-1}\right)$.

Theorem 4.2 can be used in order to compute the minimum detectable input sensor fault.

Theorem 4.2: (Minimum detectable input sensor fault) Given a dynamical model (4) and monitoring the system using the observer (49), the minimum detectable input sensor fault can be computed as

$$
\begin{aligned}
\bar{f}_{u_{j, \infty}} & =\max \bar{f}_{u_{j i, \infty}}, & \bar{f}_{u_{j i, \infty}} & =+2 \frac{\left\|\left(I-C_{i} \xi_{2_{i}}(1) K_{i}\right)\left(C_{i} \xi_{1}(1) E_{\omega_{i}}+E_{v_{i}}\right)\right\|_{1}}{\left\|-C_{i j} \xi_{2_{i j}}(1) B_{i j} F_{u_{i j}}\right\|_{1}}, \\
\underline{f}_{u_{j, \infty}} & =\min \underline{f}_{u_{j i, \infty}}, & \underline{f}_{u_{j i, \infty}} & =-2 \frac{\left\|\left(I-C_{i} \xi_{2_{i}}(1) K_{i}\right)\left(C_{i} \xi_{1}(1) E_{\omega_{i}}+E_{v_{i}}\right)\right\|_{1}}{\left\|-C_{i j} \xi_{2_{i j}}(1) B_{i j} F_{u_{i j}}\right\|_{1}},
\end{aligned}
$$

where $\bar{f}_{u_{j, \infty}}$ and $\underline{f}_{u_{j, \infty}}$ are upper and lower bounds of the minimum detectable input sensor fault, respectively.

Proof. As mentioned in the proof of Theorem 4.1, in the faulty scenario, i.e., $f_{u}\left(q^{-1}\right) \neq 0 \Rightarrow$ $f_{u_{\infty}} \neq 0$, the condition (46) can be written considering (53) and based on Proposition 3.6. Thus, in the faulty condition (input sensor fault) the satisfaction of the following condition should be proved $\forall i, j$ :

$$
\mathscr{G}_{f_{u_{i j}}}(1) f_{u_{j, \infty}} \notin 0 \pm 2\left\|\mathscr{G}_{\omega_{i}}(1)+\mathscr{G}_{v_{i}}(1)\right\|_{1}
$$


Then, the MDF can be computed using (56) as

$$
f_{u_{j, \infty}} \notin 0 \pm 2 \frac{\left\|\mathscr{G}_{\omega_{i}}(1)+\mathscr{G}_{v_{i}}(1)\right\|_{1}}{\left\|\mathscr{G}_{f_{u_{i j}}}(1)\right\|_{1}} .
$$

Hence, the same formulation as (55) is obtained by substituting (35b), (35c) and (54) in (57) for the sensitivity with respect to $\omega, v$ and $f_{u}$, respectively.

\subsection{Minimum detectable actuator fault}

The dynamical model including an actuator fault can be written as

$$
\begin{aligned}
x_{+} & =A x+B u+E_{\omega} \omega+F_{a} f_{a}, \\
y & =C x+E_{v} v
\end{aligned}
$$

where $f_{a}$ denotes the actuator fault and $F_{a}$ is the associated matrix of the given fault. In addition, $F_{a}$ indicates the actuator that is affected by the fault with suitable dimension as

$$
F_{a} \in \mathbb{R}^{n_{x} \times n_{u}} .
$$

Furthermore, the measurement equation in (58) can be expressed in input-output form using the shift operator $q^{-1}$ as

$$
y=\mathscr{H}_{u}\left(q^{-1}\right) u+\mathscr{H}_{\omega}\left(q^{-1}\right) \omega+\mathscr{H}_{v}\left(q^{-1}\right) v+\mathscr{H}_{f_{a}}\left(q^{-1}\right) f_{a},
$$

where

$$
\mathscr{H}_{f_{a}}\left(q^{-1}\right)=C \xi_{1}\left(q^{-1}\right) F_{a} .
$$

On the other hand, the observer (5) can be used for monitoring the dynamical model (58), which can be expressed in input-output form as (32). Then, the residual in the case of the actuator fault in input-output form can be written as

$$
r=\mathscr{G}_{u}\left(q^{-1}\right) u+\mathscr{G}_{\omega}\left(q^{-1}\right) \omega+\mathscr{G}_{v}\left(q^{-1}\right) v+\mathscr{G}_{f_{a}}\left(q^{-1}\right) f_{a},
$$

with

$$
\mathscr{G}_{f_{a}}\left(q^{-1}\right)=\left(I-\mathscr{T}_{y}\left(q^{-1}\right)\right) \mathscr{H}_{f_{a}}\left(q^{-1}\right)
$$

where $\mathscr{G}_{f_{a}}$ denotes the sensitivity of the residual with respect to the actuator fault. Moreover, $\mathscr{G}_{\omega}$ and $\mathscr{G}_{v}$ indicate the sensitivity of the residual with respect to the disturbance $\omega$ and the measurement noise $v$, respectively.

Theorem 4.3 can be used in order to compute the minimum detectable actuator fault.

Theorem 4.3: (Minimum detectable actuator fault) Considering a dynamical model (58) and the 
Table 1.: Residual sensitivity with respect to different type of actuator and sensor faults.

\begin{tabular}{ll}
\hline & Residual sensitivity \\
\hline Output sensor fault & $\mathscr{G}_{f_{y}}\left(q^{-1}\right)=\left(I-C \xi_{2}\left(q^{-1}\right) K\right) F_{y}$ \\
Input sensor fault & $\mathscr{G}_{f_{u}}\left(q^{-1}\right)=-C \xi_{2}\left(q^{-1}\right) B F_{u}$ \\
Actuator fault & $\mathscr{G}_{f_{a}}\left(q^{-1}\right)=\left(I-C \xi_{2}\left(q^{-1}\right) K\right) C \xi_{1}\left(q^{-1}\right) F_{a}$ \\
\hline
\end{tabular}

observer in (5), the minimum detectable actuator fault can be computed as

$$
\begin{aligned}
\bar{f}_{a_{j, \infty}} & =\max \bar{f}_{a_{j i, \infty}}, & \bar{f}_{a_{j i, \infty}} & =+2 \frac{\left\|\left(I-C_{i} \xi_{2_{i}}(1) K_{i}\right)\left(C_{i} \xi_{1}(1) E_{\omega_{i}}+E_{v_{i}}\right)\right\|_{1}}{\left\|\left(I-C_{i j} \xi_{2_{i j}}(1) K_{i j}\right) C_{i j} \xi_{1_{i j}}(1) F_{a_{i j}}\right\|_{1}} \\
\underline{f}_{a_{j, \infty}} & =\min \underline{f}_{a_{j i, \infty}}, & \underline{f}_{a_{j i, \infty}} & =-2 \frac{\left\|\left(I-C_{i} \xi_{2_{i}}(1) K_{i}\right)\left(C_{i} \xi_{1}(1) E_{\omega_{i}}+E_{v_{i}}\right)\right\|_{1}}{\left\|\left(I-C_{i j} \xi_{2_{i j}}(1) K_{i j}\right) C_{i j} \xi_{1_{i j}}(1) F_{a_{i j}}\right\|_{1}}
\end{aligned}
$$

where $\bar{f}_{a_{j, \infty}}$ and $\underline{f}_{a_{j, \infty}}$ are upper and lower bounds of the minimum detectable actuator fault, respectively.

Proof. The way of obtaining the proof is similar to those used for Theorems (4.1) and (4.2). When considering faulty scenario, i.e., $f_{a}\left(q^{-1}\right) \neq 0 \Rightarrow f_{a_{\infty}} \neq 0$, using the residual (61) and based on Proposition 3.6, the condition (46) should be satisfied and written $\forall i, j$ as

$$
\mathscr{G}_{f_{a_{i j}}}(1) f_{a_{j, \infty}} \notin 0 \pm 2\left\|\mathscr{G}_{\omega_{i}}(1)+\mathscr{G}_{v_{i}}(1)\right\|_{1} .
$$

Now, simplifying (64) yields

$$
f_{a_{j, \infty}} \notin 0 \pm 2 \frac{\left\|\mathscr{G}_{\omega_{i}}(1)+\mathscr{G}_{i_{i}}(1)\right\|_{1}}{\left\|\mathscr{G}_{f_{a_{i j}}}(1)\right\|_{1}} .
$$

Therefore, the minimum detectable actuator fault can be formulated as (63) by substituting (35b), $(35 \mathrm{c})$ and $(62)$ in (65).

\subsection{Comparative assessment}

This section attempts to find the bridge between the approaches characterizing the MDF undertaken by the classical sensitivity framework using interval observers and the set-invariance approach. In this regard, according to (Blanke et al., 2006), the considered faults are classified into different categories depending on its location as

- actuator faults, which affect the system inputs,

- sensor faults that affect the measurements of the inputs and outputs of the system.

As a result of considering different mentioned actuator and sensors faults, the sensitivity of the residual with respect to the fault is computed differently. Table 1 summarizes the derived formulation for different sensitivity in previous sections.

Consequently, the characterized formulation for computing the minimum magnitude of the given fault is different. Table 2 summarizes the characterization of the MDF for different type of considered faults. 
Table 2.: Different minimum detectable actuator and sensor faults.

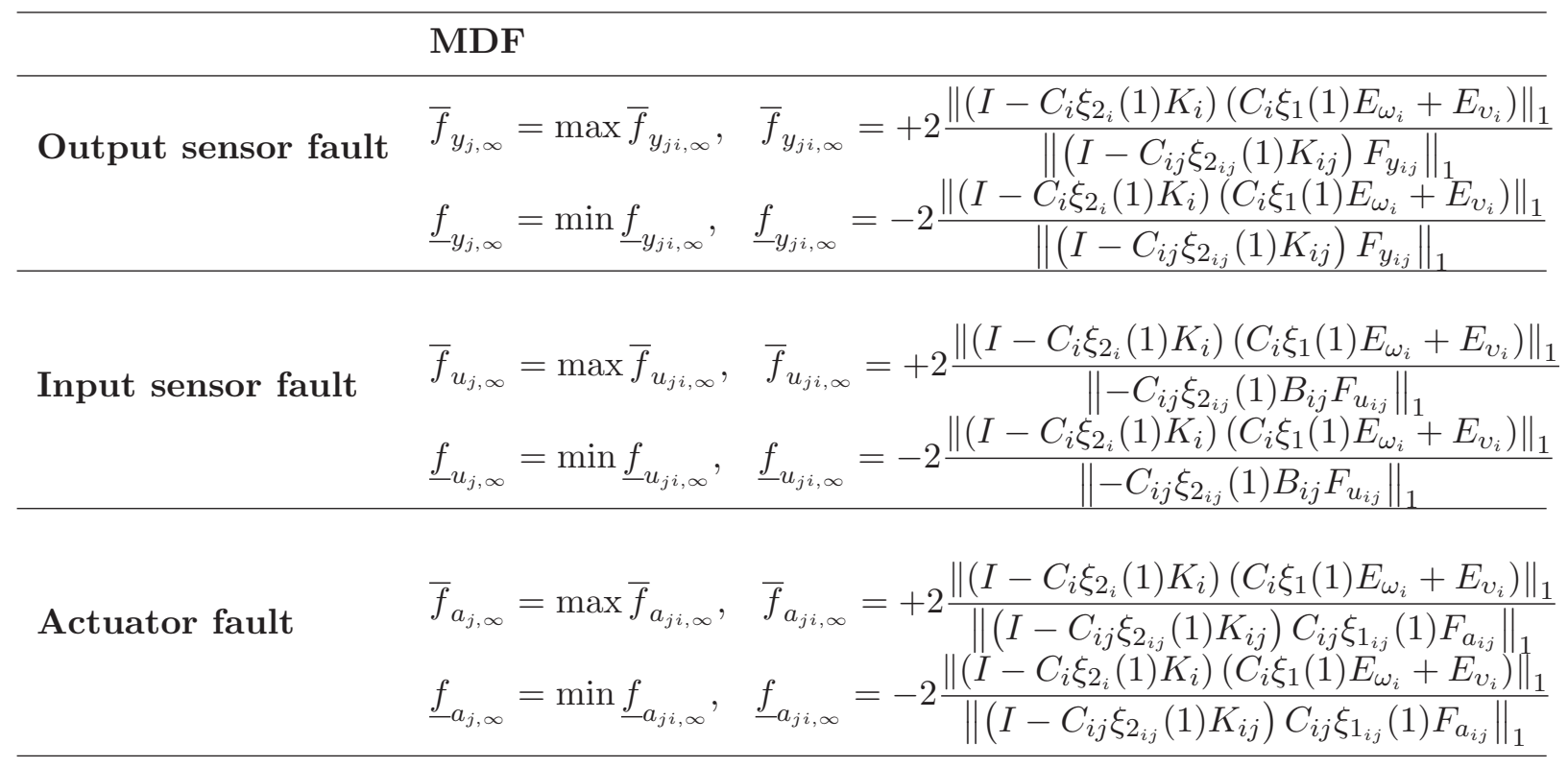

Further analysis on the physical meaning of the different type of the considered faults will be discussed in Section 5 based on a case study.

\section{Case study}

\subsection{General description}

The MDF analysis is performed using a quadruple-tank system based on the well-known benchmark proposed in (Johansson, 2000). A schematic of the system can be seen in Figure 2.

As shown in Figure 2, the two system inputs are the pump flows that are determined when applying voltage $v_{1}$ and $v_{2}$ to the pump which can be manipulated to regulate the tanks level. Thus, the action of the pumps is to pour the tanks by the extraction of the water from the basin. Moreover, Tanks 1 and 2 are located below of Tanks 3 and 4 . Thus, the lower tanks receive additional water flow from the upper tanks by gravity. Furthermore, the water levels in Tanks 1 and 2 are the output of the system which are obtained as voltages from the measurement devices between $0 \mathrm{~V}$ to $10 \mathrm{~V}$. Moreover, the input of each tank is affected by the aperture of the valve, which can vary between 0 and 1 , i.e., $0 \leq \gamma_{j} \leq 1$ with $i=1,2$.

Regarding to the psychical features, the tank are made from plexiglas tubes with the height of $20 \mathrm{~cm}$. Also, the connection of the tanks and the pumps is done using the pipe with a diameter 6 $\mathrm{mm}$.

From the mathematical point of view, the following nonlinear differential equations describe the dynamics of the considered system, which can be written using mass-balance relations and 


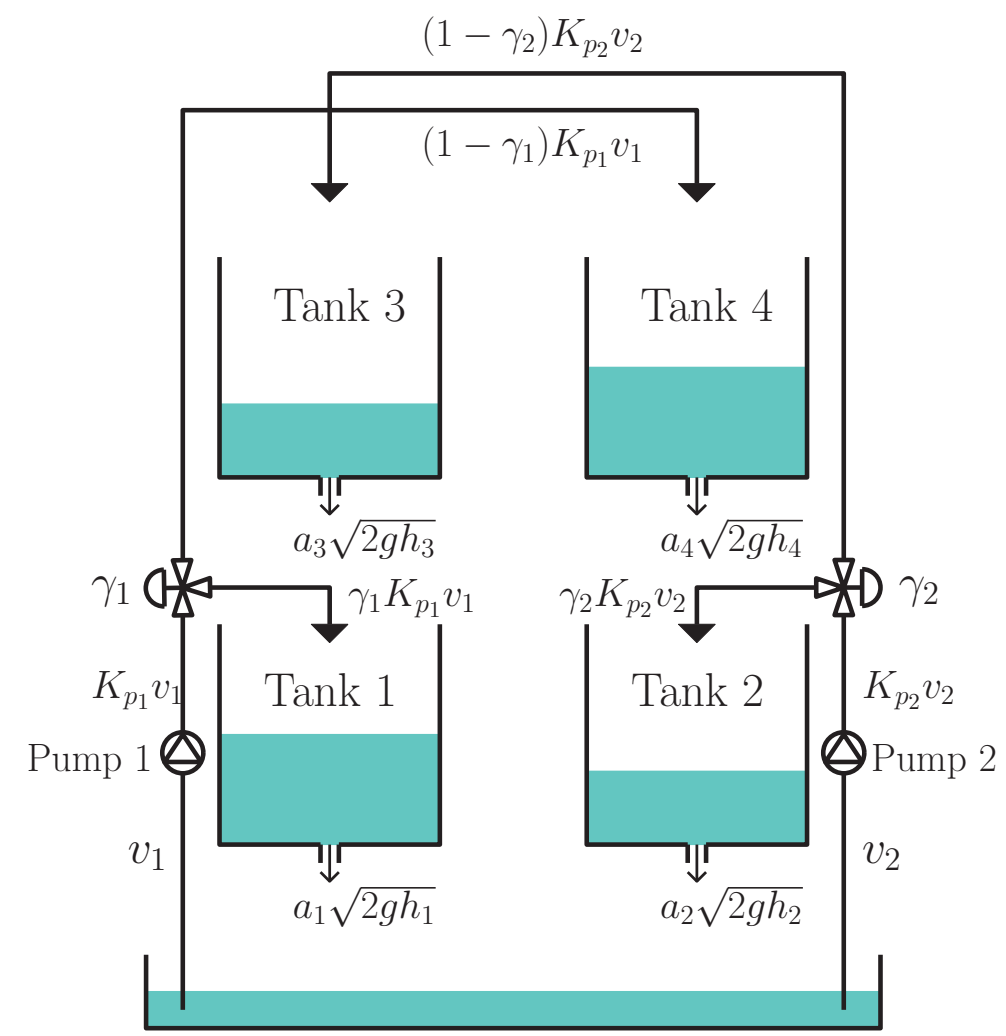

Figure 2.: Schematic diagram of the quadruple-tank system.

Bernoulli's law:

$$
\begin{aligned}
& \frac{d h_{1}(t)}{d t}=-\frac{a_{1}}{A_{1}} \sqrt{2 g h_{1}(t)}+\frac{a_{3}}{A_{1}} \sqrt{2 g h_{3}(t)}+\frac{\gamma_{1} K_{p_{1}}}{A_{1}} v_{1}(t), \\
& \frac{d h_{2}(t)}{d t}=-\frac{a_{2}}{A_{2}} \sqrt{2 g h_{2}(t)}+\frac{a_{4}}{A_{2}} \sqrt{2 g h_{4}(t)}+\frac{\gamma_{2} K_{p_{2}}}{A_{2}} v(t), \\
& \frac{d h_{3}(t)}{d t}=-\frac{a_{3}}{A_{3}} \sqrt{2 g h_{3}(t)}+\frac{\left(1-\gamma_{2}\right) K_{p_{2}}}{A_{3}} v_{2}(t), \\
& \frac{d h_{4}(t)}{d t}=-\frac{a_{4}}{A_{4}} \sqrt{2 g h_{4}(t)}+\frac{\left(1-\gamma_{1}\right) K_{p_{1}}}{A_{4}} v_{1}(t),
\end{aligned}
$$

where $v_{j}$ denotes the input voltage through the Pump $j$ with $j=1,2$ and $h_{i}$ is the water level in Tank $i$ with $i=1,2,3,4$. Moreover, $K_{p_{i}}$ is the constant of pump $j$ and $g$ is the acceleration due to gravity. According Johansson (2000), the parameter values in (66) are

- $A_{1}=A_{3}=28 \mathrm{~cm}^{2}$,

- $A_{2}=A_{4}=32 \mathrm{~cm}^{2}$,

- $a_{1}=a_{3}=0.071 \mathrm{~cm}^{2}$,
- $a_{2}=a_{4}=0.057 \mathrm{~cm}^{2}$,

- $\gamma_{j}=0.60$,

- $g=981 \mathrm{~cm} / \mathrm{s}^{2}$.

Since all the mathematical developments in previous sections were obtained based on the consideration of a linear dynamic system, the linearized model of (66) is required to be obtained to illustrate the effectiveness of the characterized formulations. In this regard, the model (66) is linearized around a working point. The selection of the operating point is done when the system exhibits minimum-phase characteristics. The operating point is denoted using the superscript * and selected as 
- $\left(h_{1}^{*}, h_{2}^{*}\right) \quad=(12.4,12.7) \mathrm{cm}$,

- $\left(h_{3}^{*}, h_{4}^{*}\right) \quad=(1.8,1.4) \mathrm{cm}$,

- $\left(v_{1}^{*}, v_{2}^{*}\right) \quad=(3,3) \mathrm{V}$,

- $\left(K_{p_{1}}^{*}, K_{p_{2}}^{*}\right)=(3.33,3.35) \mathrm{cm}^{3} / \mathrm{Vs}$,

- $\left(\gamma_{1}^{*}, \gamma_{2}^{*}\right)=(0.7,0.6)$.

Therefore, the linearized model of (66) can be written by introducing the variables $\tilde{h}_{i}=h_{i}-h_{i}^{*}$ and $\tilde{v}_{i}=v_{i}-v_{i}^{*}$ as

$$
\begin{aligned}
& \dot{\tilde{h}}(t)=\left[\begin{array}{cccc}
-\frac{1}{T_{1}} & 0 & \frac{A_{3}}{A_{1} T_{3}} & 0 \\
0 & -\frac{1}{T_{2}} & 0 & \frac{A_{3}}{A_{1} T_{3}} \\
0 & 0 & -\frac{1}{T_{3}} & 0 \\
0 & 0 & 0 & -\frac{1}{T_{4}}
\end{array}\right] \tilde{h}(t)+\left[\begin{array}{cc}
\frac{\gamma_{1} K_{p_{1}}}{A_{1}} & 0 \\
0 & \frac{\gamma_{2} K_{p_{2}}}{A_{2}} \\
0 & \frac{\left(1-\gamma_{1}\right) K_{p_{2}}}{A_{3}} \\
\frac{\left(1-\gamma_{1}\right) K_{p_{1}}}{A_{4}} & 0
\end{array}\right] \tilde{v}(t), \\
& y(t)=\left[\begin{array}{cccc}
K_{c} & 0 & 0 & 0 \\
0 & K_{c} & 0 & 0
\end{array}\right] \tilde{h}(t),
\end{aligned}
$$

where $T_{i}=\frac{A_{i}}{a_{i}} \sqrt{\frac{2 h_{i}^{*}}{g}}$, with $i=1,2,3,4$ and $K_{c} h(t)$ shows the measured level signal that is determined from the level measurement device. Then, considering $K_{c}=0.50 \mathrm{~V} / \mathrm{cm}$ according to Johansson (2000), the range of the measured output is $10 \mathrm{~V}$ since the height of each tank is $20 \mathrm{~cm}$. In addition, the incremental value of the measured output around the operation point of Tank 1 is from $8 \mathrm{~cm}$ to $16 \mathrm{~cm}$ or from $4 \mathrm{~V}$ to $8 \mathrm{~V}$.

Finally, using the Euler discretization with a sampling time of $1 \mathrm{~s}$, the discrete-time state-space model of the considered two-tank system affected by the additional disturbance and noise can be written as

$$
\begin{aligned}
\tilde{h}_{+} & =A \tilde{h}+B \tilde{v}+E_{\omega} \omega, \\
y & =C \tilde{h}+D \tilde{v}+E_{v} v,
\end{aligned}
$$

$A=\left[\begin{array}{cccc}0.9842 & 0 & 0.0419 & 0 \\ 0 & 0.9890 & 0 & 0.0333 \\ 0 & 0 & 0.9581 & 0 \\ 0 & 0 & 0 & 0.9672\end{array}\right], \quad B=\left[\begin{array}{cc}0.2102 & 0 \\ 0 & 0.0628 \\ 0 & 0.0479 \\ 0.0094 & 0\end{array}\right], \quad C=\left[\begin{array}{cccc}0.5 & 0 & 0 & 0 \\ 0 & 0.5 & 0 & 0\end{array}\right], \quad D=\left[\begin{array}{ll}0 & 0 \\ 0 & 0\end{array}\right]$

Moreover, bounded disturbances influencing all the state-space directions and the measurement noise are modeled respectively with $E_{\omega}$ and $E_{v}$ in (68) as

$$
E_{\omega}=\left[\begin{array}{ccccccc}
0.05 & 0.01 & 0 & 0 & 0 & 0 & 0 \\
0.05 & 0 & 0.01 & 0 & 0 & 0 & 0 \\
0.05 & 0 & 0 & 0.01 & 0 & 0 & 0 \\
0.05 & 0 & 0 & 0 & 0.01 & 0 & 0
\end{array}\right], \quad E_{v}=\left[\begin{array}{ll}
0_{2 x 5} & 0.1 I_{2}
\end{array}\right] .
$$

Then, the effect of the fault is modeled through the selection of matrix $F_{\bullet}$ where the subscript can be assigned by $y, u$ and $a$ depending on the kind of fault considered (see (40), (49) and (58)).

Furthermore, following the problem formulation in Section 2, both state and measurement uncertainty vectors, i.e., $\omega$ and $v$, and all the considered fault vectors, i.e., $f_{a}, f_{y}$ and $f_{u}$, are assumed 
to be normalized in $[-1,1]$. Accordingly, the matrices $E_{\omega}, E_{v}, F_{a}, F_{y}$ and $F_{u}$ are defined. Furthermore, the observer gain is computed based on the proposed method in (Combastel, 2015).

In this regard, further investigation will be conducted for the case study by considering the single additive actuator and sensors faults to compute the minimum detectable magnitude in the next section.

\subsection{Minimum Detectable Faults (MDF) analysis}

In this section, the actuator and the sensor faults are considered for the case study as single additive fault, separately. First, the magnitude of MDF is computed based on the theoretical formulation that are derived in Section 4. Then, the simulation is employed in order to validate the obtained results.

\subsubsection{Minimum detectable actuator fault}

The actuator is electrically driven allowing to manipulate the valve. Moreover, the valve actuator can be either open or closed and intermediate positions. As it is mentioned in the description of the case study, the range of flow parameter is considered between 0 and 1 . This parameter is related to the position of the valve during the experiment and the flow to the lower and upper tanks are affected by the position of the valve through $\gamma_{j} K_{p_{j}} v_{j}$ and $\left(I-\gamma_{j}\right) K_{p_{j}} v_{j}$ with $j=1,2$, respectively.

Furthermore, the effect of actuator fault can be simulated based on (faulty) dynamical model (58) through matrix $F_{a}$ and the vector $f_{a}$. In this case, single actuator fault is considered based on the elements of the vector $f_{a}$, i.e.,

$$
f_{a}=\left[\begin{array}{l}
f_{a_{1}} \\
f_{a_{2}}
\end{array}\right],
$$

where $f_{a_{1}}$ and $f_{a_{2}}$ indicate the fault affecting each actuator. Furthermore, the following matrix $F_{a}$ is selected to simulate the actuator fault:

$$
F_{a}=5 B .
$$

All the previously mentioned points are considered to simulate the actuator fault for the case study. Then, considering Theorem 4.3, the minimum magnitude of the actuator fault that can be detected is obtained in steady state as

$$
\begin{aligned}
& f_{a_{1}}= \pm 0.0879 \\
& f_{a_{2}}= \pm 0.4095
\end{aligned}
$$

In this case study, the flow parameters are considered as $\gamma_{1}=0.7$ and $\gamma_{2}=0.6$. As it can be seen in (73), the magnitude of the minimum actuator fault that can be detected is \pm 0.0879 for $f_{1}$ and \pm 0.4095 for $f_{2}$. It means that when the magnitude of the actuator fault is higher than the magnitudes obtained in (73) can be detected. Otherwise, it is not detectable. Using in this case, when $\gamma_{1}>0.7 \pm 0.0879$ or $\gamma_{2}>0.6 \pm 0.4095$, the fault is detected.

Furthermore, the size of the MDF is obtained based on the simulation by increasing the magnitude of the actuator fault from 0 until the magnitude that can be detected at the end of the simulation, i.e., steady state. The following magnitude are obtained from the simulation:

$$
\begin{aligned}
& f_{a_{1}}^{\circ}= \pm 0.0650 \\
& f_{a_{2}}^{\circ}= \pm 0.3800,
\end{aligned}
$$


where the superscript $\circ$ shows the magnitude is obtained from the simulation.

Now by comparing (73) with (74), there is no significant difference between the obtained MDF using the theoretical approach and the simulation is found. Thus, (74) confirms the obtained magnitude in (73) using Theorem 4.3.

For the goal of further analysis, the magnitude of fault $f_{2}$ in the pump valve is considered as a single step additive fault by the following scenarios in order to show the FD performance:

- slightly bigger than 0.4095,

- slightly smaller than 0.4095 .
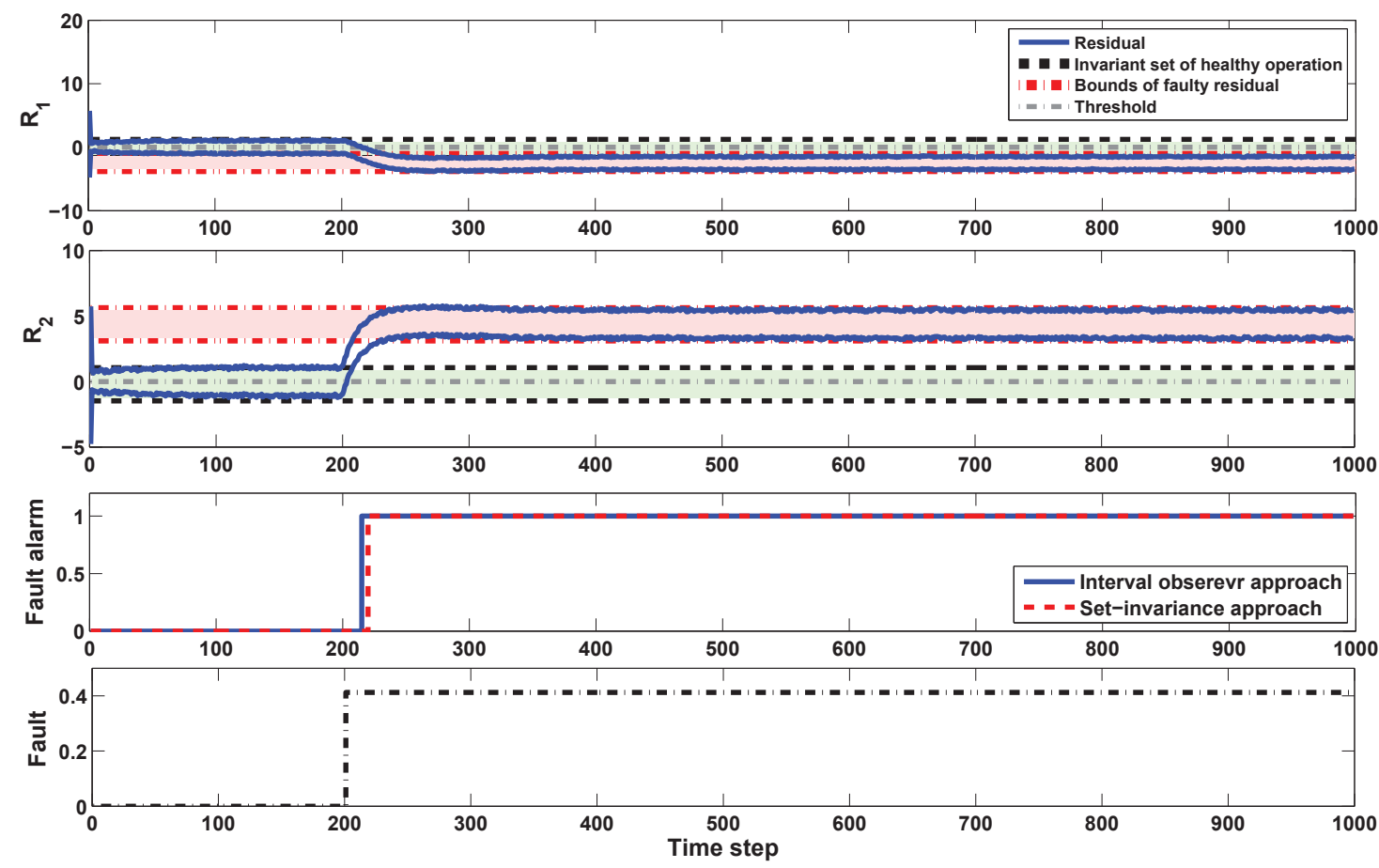

Figure 3.: FD test in the case of actuator fault with the magnitude bigger than 0.4095.

In Figure 3, the obtained residuals from the simulation are shown in the case that the position of the $\gamma_{2}$ is considered as a faulty parameter with $\gamma_{2}>0.6 \pm 0.4095$ and $\gamma_{1}$ is considered healthy in steady state. Precisely, the fault is added to the valve actuator through the dynamical model (58) at $k=200$ with the magnitude bigger than 0.4095 for the case of actuator fault. In addition, the projection of the invariant residual sets that are generated based on the healthy mode of the system (considering both outputs) is shown in Figure 3.

As seen in Figure 3, from time step $k=0$ until $k=200$, both actuators are healthy and working with $\gamma_{1}=0.7$ and $\gamma_{2}=0.6$. Thus, the generated residuals are inside the healthy invariant sets. On the other hand, the residual sets changes at $k=200$ due to the occurrence of the fault and go outside of their obtained invariant sets.

At this point, that the interval observer-based approach and set-invariance theory can be compared in the proposed FD framework. As it can be seen in Figure 3, using the interval observer-based approach, the fault can be detected sooner since, according to Algorithm 1, FD test considering the interval observer-based approach is done only by cheeking if $0 \notin\left\langle c_{r^{i o}}, b\left(R_{r^{i o}}\right)\right\rangle$. But, based on the set-invariance theory, the existence of the fault is proved when the residual (that is determined iteratively at each time instant) satisfies $r \notin\left\langle c_{r_{\infty}^{i s}}, R_{r_{\infty}^{i s}}^{i s}\right\rangle$. Thus, moving the residual from the healthy set to the faulty residual set can take more time in comparison with only cheeking if $0 \notin\left\langle c_{r^{i o}}, b\left(R_{r^{i o}}\right)\right\rangle$. Therefore, the existence of the fault is shown latter. What is interesting from 
Figure 3 is that, by considering the proposed FD approach, both interval observer-based approach and set-invariance theory are able to detect the fault with the magnitude slightly bigger than 0.4095 (bottom plot in Figure 3 shows the applied fault magnitude). The FD decision is indicated by 0 in case of no-fault in the system and 1 indicating that the fault is detected.

Considering Remark 1, the computational burden of the interval observer-based approach comes from the type of the considered sets (here zonotopes) in order to bound the effect of uncertainties. As it was mentioned before, the computationally efficient way to implement the FD test without increasing the false alarm rate is to check whether or not 0 belongs to an aligned box enclosing the zonotope $\left\langle c_{\text {rio }}, R_{r^{i o}}\right\rangle$. In this regard, Figure 4 compares the runtime of the FD test considering $0 \notin\left\langle c_{r^{i o}}, b\left(R_{r^{i o}}\right)\right\rangle$ (known as interval observer-based approach) versus the FD test using the exact zonotope (known as zonotopic observer-based approach) at each time instant. Furthermore, Table 3 compares the runtime for considering the whole time range of the simulation. It can be observed from both Figure 4 and Table 3 that the computational burden when using the aligned box enclosing the zonotope $\left\langle c_{r^{i o}}, R_{r^{i o}}\right\rangle$ for detecting the fault is less than the case of using the exact zonotope.

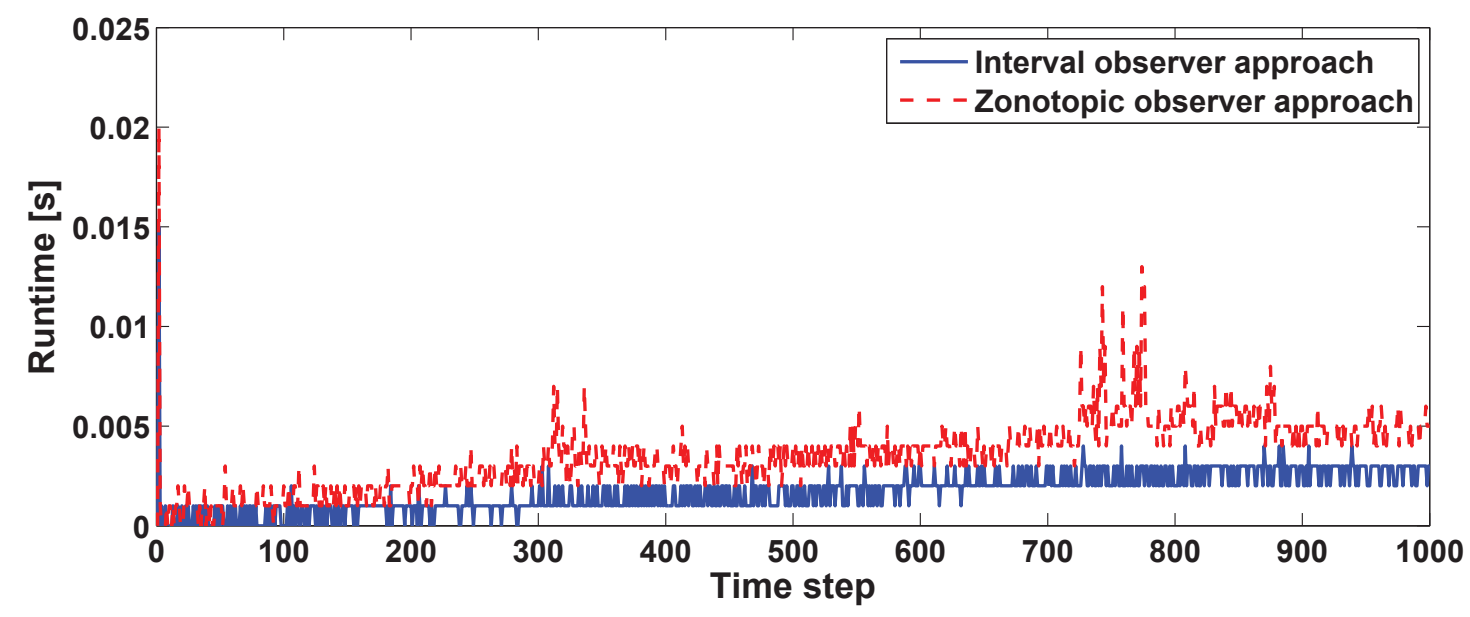

Figure 4.: Runtime comparison using interval and zonotopic observer-based approaches at each time step.

Table 3.: Runtime comparison using interval and zonotopic observer-based approaches considering the whole time range of the simulation.

\begin{tabular}{lc}
\hline & Runtime $[\mathrm{s}]$ \\
\hline Interval observer-based approach & 1.6240 \\
Zonotopic observer-based approach & 3.0430 \\
\hline
\end{tabular}

Likewise, the FD test is carried out in the case that the magnitude of the actuator fault is slightly smaller than 0.4095, fact that is shown in bottom plot of Figure 5. This figure shows that the fault is not detectable for both set-invariance theory and interval observer-based approach since neither Algorithm 1 nor (25) are satisfied. Therefore, the fault with the magnitude smaller than 0.4095 cannot be detected.

\subsubsection{Minimum detectable output sensor fault}

The MDF analysis in the case of output sensor fault can be done based on Theorem 4.1. As it is mentioned, the outputs of the quadruple-tank system are the water levels in Tanks 1 and 2 that 

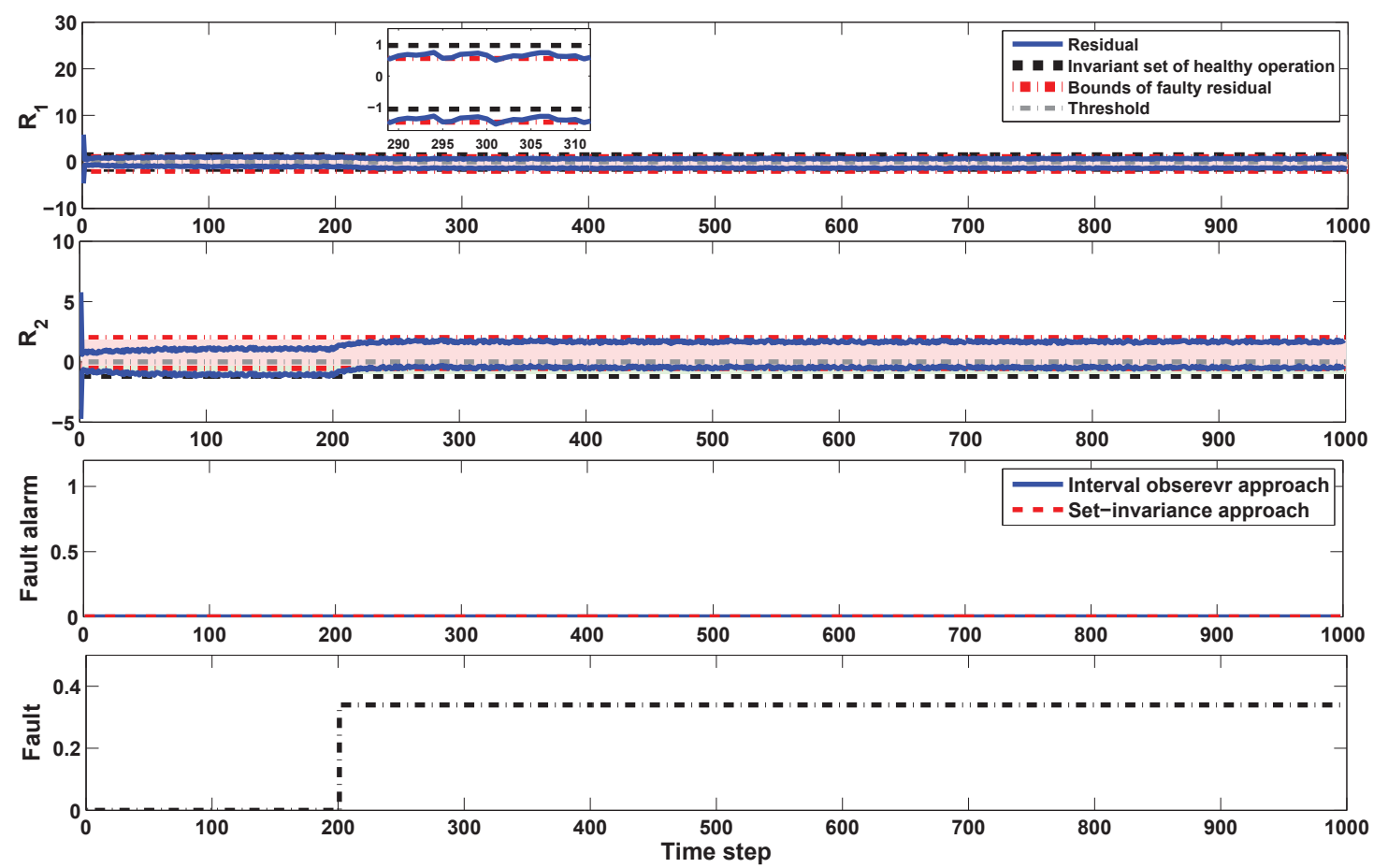

Figure 5.: FD test in the case of actuator fault with the magnitude smaller than 0.4095.

are measured as voltages from the measurement devices. In this case study, the height of each tank is $20 \mathrm{~cm}$. Then, each output of the system is between $0-10 \mathrm{~V}$ since $K_{c}=0.50 \mathrm{~V} / \mathrm{cm}$.

In this case, the fault is simulated using the dynamical model (40) and the element of vector $f_{y}$, i.e.,

$$
f_{y}=\left[\begin{array}{l}
f_{y_{1}} \\
f_{y_{2}}
\end{array}\right],
$$

where $f_{y_{1}}$ and $f_{y_{2}}$ show the effect of the fault influencing each output. On the other hand, matrix $F_{y}$ is defined with the whole range of the measurement as

$$
F_{y}=\left[\begin{array}{cc}
10 & 0 \\
0 & 10
\end{array}\right] .
$$

Turning now to the main goal of this section, the minimum detectable output sensor fault can be determined in steady state using (45) based on Theorem 4.1 as

$$
\begin{aligned}
& f_{y_{1}}= \pm 0.2808 \mathrm{~V}, \\
& f_{y_{2}}= \pm 0.5710 \mathrm{~V} .
\end{aligned}
$$

Thus, it can be realized that the magnitudes of the output sensor fault bigger than $0.2808 \mathrm{~V}$ for $f_{y_{1}}$ and $0.5710 \mathrm{~V}$ for $f_{y_{2}}$ is detectable in steady state. In this case, the operating points that are considered for the water levels of the Tanks 1 and 2 are around $12.4 \mathrm{~cm}$ (or $6.2 \mathrm{~V}$ ) and 12.7 (or $6.35 \mathrm{~V})$, respectively. Thus, the existence of the fault can be detected on each sensor, in the case that its magnitude is bigger magnitude than the obtained results in (77).

Moreover, the following magnitude of MDF is obtained based on the simulation by increasing the size of the output sensor fault until the magnitude that can be detected in steady state, i.e., 
at the end of simulation:

$$
\begin{aligned}
& f_{y_{1}}^{\circ}= \pm 0.2750 \mathrm{~V}, \\
& f_{y_{2}}^{\circ}= \pm 0.5650 \mathrm{~V} .
\end{aligned}
$$

The similarity of results is apparent from the comparison of the two magnitudes posted in (77) and (78) using theoretical approach and simulation to compute MDF. Therefore, (78) can be considered as a validation of (77) which is obtained based on Theorem 4.1.
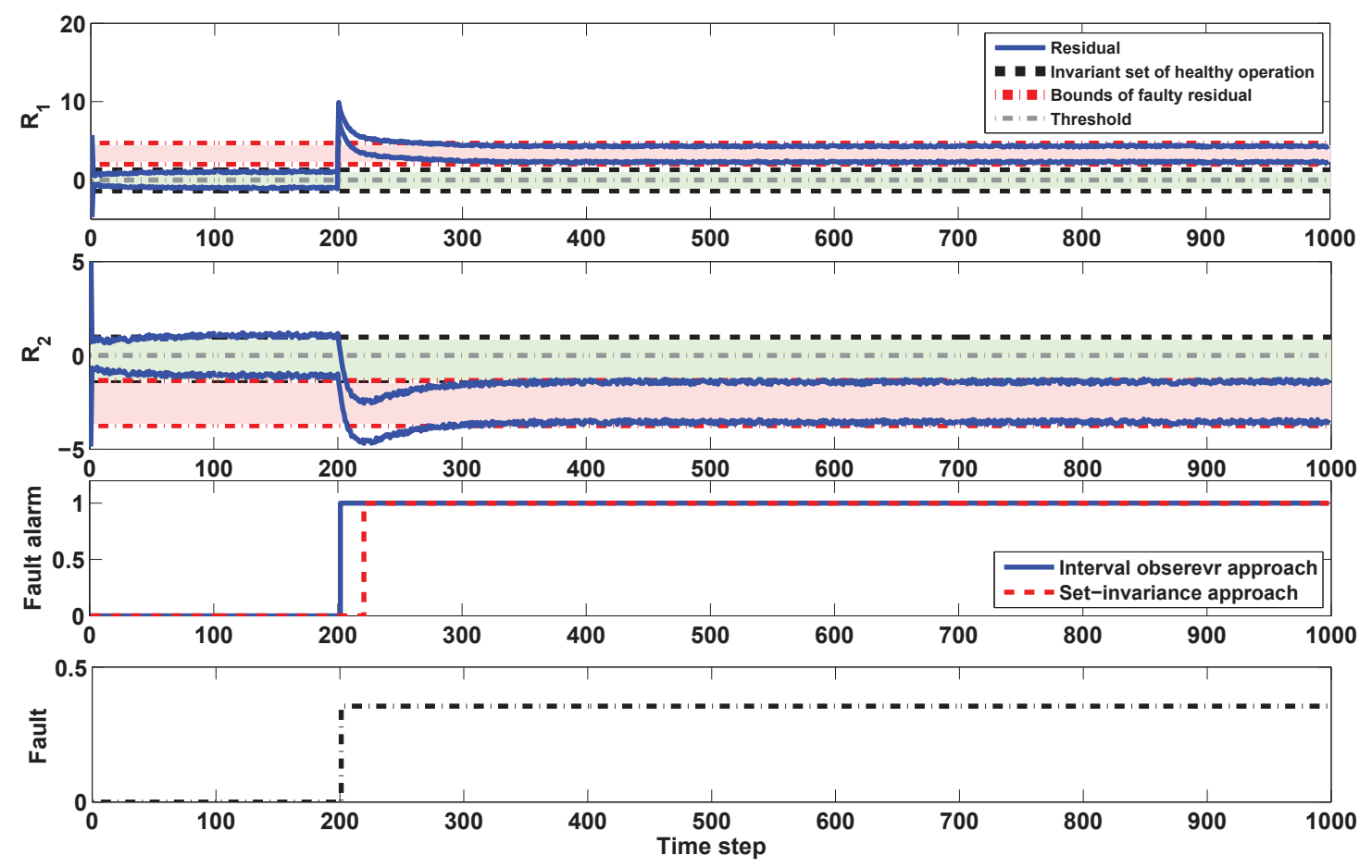

Figure 6.: FD test in the case of output sensor fault with the magnitude bigger than $0.2808 \mathrm{~V}$.

Regarding the FD performance, the following scenarios are considered when one of the output measured voltages from the level measurement devices (e.g., $f_{y_{1}}$ ) is influenced by a single additive step fault:

- slightly bigger than $0.2808 \mathrm{~V}$,

- slightly smaller than $0.2808 \mathrm{~V}$.

The output sensor fault is simulated for the case study from $k=200$ until the end of the simulation and the FD test presented in Section 3 is used in order to show the FD performance. In the first scenario, the FD test is applied when the magnitude of the fault is slightly bigger than $0.2808 \mathrm{~V}$. Figure 6 shows the obtained results from the simulation of the first scenario.

As can be seen from Figure 6, the system is working properly until $k=200$ since the residual is inside of the healthy invariant set. But, after the occurrence of the fault at $k=200$, the residuals are effected by the given fault and the empty intersection can be found between the residual sets and the healthy invariant sets. Thus, the fault alarm shows 1 that means the detection of the fault (see the bottom plot in Figure 6). The other point that is worth mentioning regarding Figure 6 is related to the comparison of interval observer-based approach and set-invariance theory from the detection time point of view. Figure 6 shows that the decision of the fault occurrence is faster using the interval observer-based approach in comparison with the set-invariance theory. As it is mentioned, the satisfaction of FD test in (25) based on set-invariance required more time than the use of Algorithm 1. This faster FD decision shows the advantage of interval observer-based 
approach in comparison with the set-invariance theory. But, the point regarding the Figure 6 is that the fault with the obtained magnitude based on the theoretical formulation is detectable for both mentioned approaches.
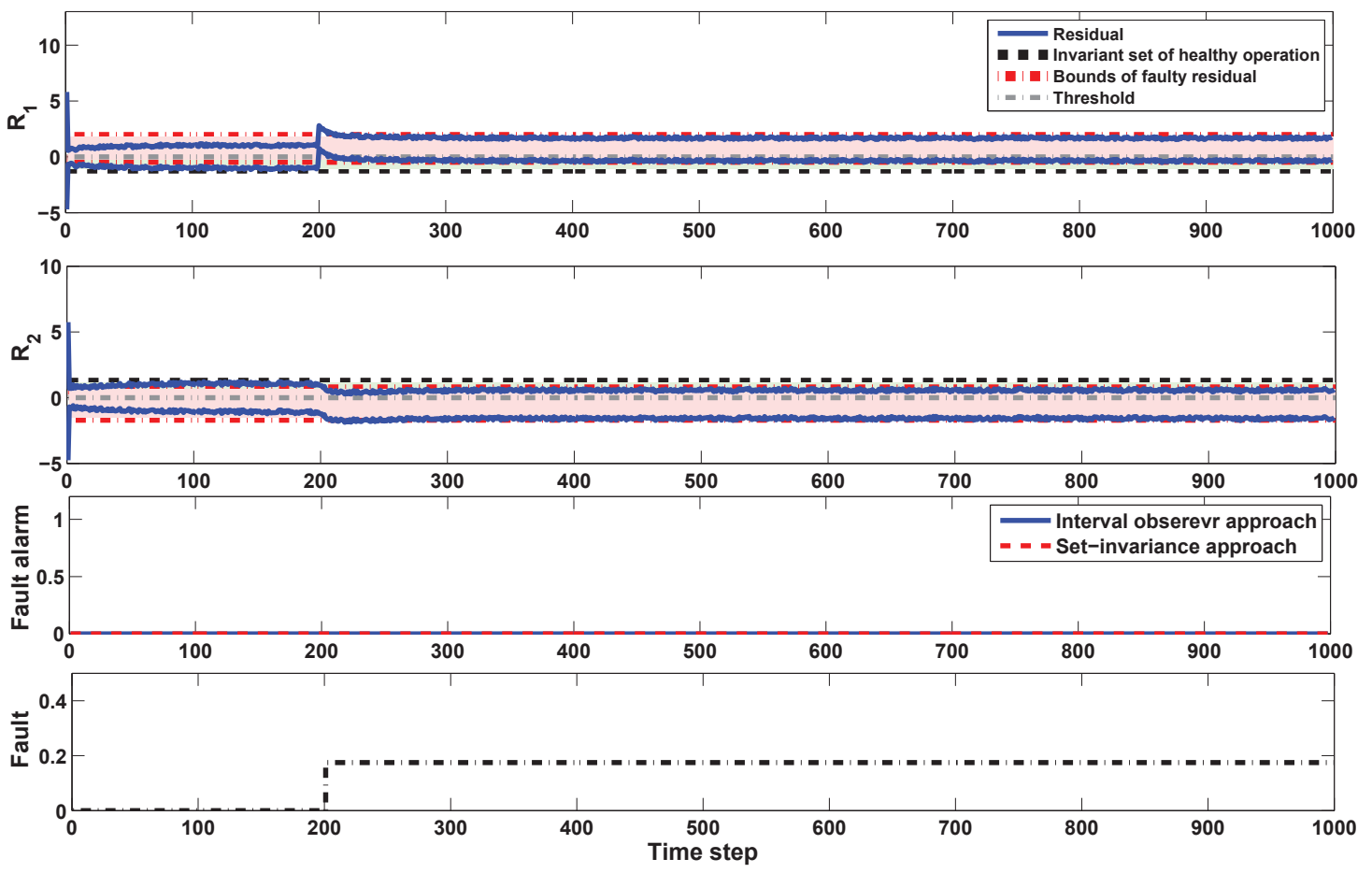

Figure 7.: FD test in the case of output sensor fault with the magnitude smaller than $0.2808 \mathrm{~V}$.

Finally, the FD test is applied to the case that a fault occurs with the magnitude slightly smaller than 0.2808 V. Figure 7 shows the simulation of this scenario.

The overlap between the residual set and the invariant set can be observed from Figure 7 . Therefore, the effect of the fault is not detectable since this overlap exists. Thus, if the output sensor fault occurs with the magnitude smaller than $0.2808 \mathrm{~V}$, the fault cannot be detected. In other words, a difference greater than $0.2808 \mathrm{~V}$ between the output measurement and its actual value can be associated with the occurrence of the fault. Therefore, the fault with the magnitude shown in bottom plot of Figure 7 is not detectable for both set-invariance theory and interval observer-based approach since neither Algorithm 1 nor (25) are satisfied.

\subsubsection{Minimum detectable input sensor fault}

Simulation of the fault is carried out through the elements of vector $f_{u}$, i.e.,

$$
f_{u}=\left[\begin{array}{l}
f_{u_{1}} \\
f_{u_{2}}
\end{array}\right]
$$

where $f_{u_{1}}$ and $f_{u_{2}}$ denote the effect of the fault influencing each observer input. Furthermore, matrix $F_{u}$ is defined with the whole range of the input as

$$
F_{u}=\left[\begin{array}{ll}
5 & 0 \\
0 & 5
\end{array}\right]
$$

Now, using all the points that are mentioned above, the minimum detectable input sensor fault 
can be theoretically obtained using (55) based on Theorem 4.2 as

$$
\begin{aligned}
& f_{u_{1}}= \pm 0.0878 \mathrm{~V}, \\
& f_{u_{2}}= \pm 0.6416 \mathrm{~V} .
\end{aligned}
$$

As mentioned before, the system input is the voltage that is linearized around $3 \mathrm{~V}$ for both pumps. Thus, based on the obtained result in (81), the fault with bigger magnitude than $0.0878 \mathrm{~V}$ influencing $f_{u_{1}}$ and $0.6416 \mathrm{~V}$ influencing $f_{u_{2}}$ is detectable. To ensure the obtained results in (81), the MDF is determined based on the simulation by increasing the magnitude of the fault from 0 $\mathrm{V}$ until the magnitude that is detectable. The following magnitude of the fault is obtained at the end of the simulation (steady state):

$$
\begin{aligned}
& f_{u_{1}}^{\circ}= \pm 0.0850 \mathrm{~V}, \\
& f_{u_{2}}^{\circ}= \pm 0.6200 \mathrm{~V} .
\end{aligned}
$$
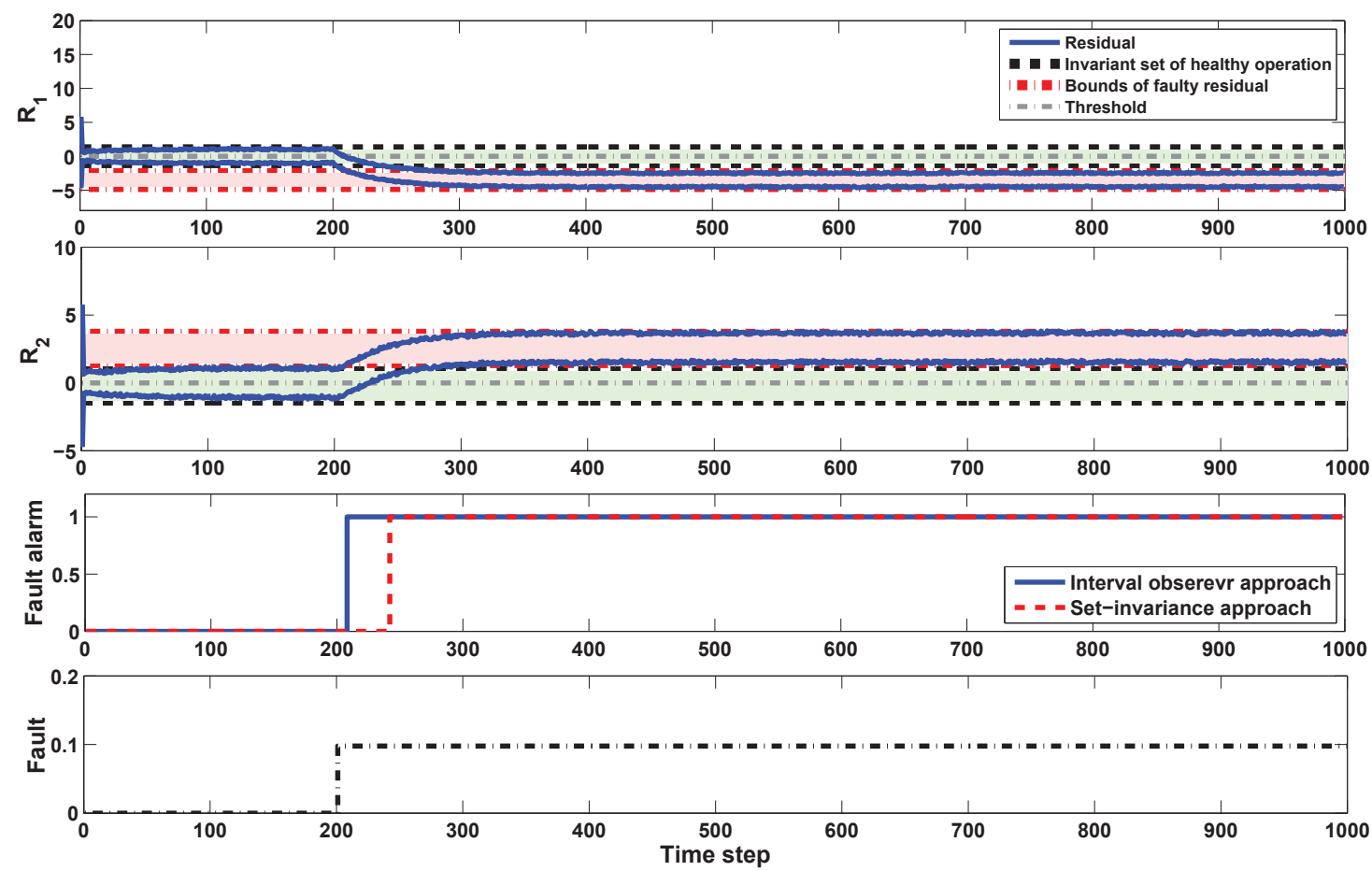

Figure 8.: FD test in the case of input sensor fault with the magnitude bigger than $0.0878 \mathrm{~V}$.

Similar to previous cases (actuator and output sensor faults), no significant difference is obtained by comparing (81), which is computed theoretically based on Theorem 4.2, and (82), which is obtained base don the simulation. Hence, (82) confirms the magnitude of the fault in (81).

A future study investigating the FD performance is done considering the following scenarios:

- slightly bigger than $0.0878 \mathrm{~V}$,

- slightly smaller than $0.0878 \mathrm{~V}$.

In this regard, Figure 8 shows the FD test in the case that the fault is considered in the input of the observer model though the pump as a single additive step fault with the magnitude slightly bigger than $0.0878 \mathrm{~V}$. Moreover, the occurrence of the fault is simulated from time instant $k=200$ and it remains until the end of the simulation.

As it can be seen from Figure 8, from time instant $k=0$ until $k=200$ both residual sets are inside of the healthy invariant sets. It means, the inputs of the system are not affected by 
the fault and consequently the system is working properly. But, after $k=200$ the residual sets moves toward outside of the healthy invariant sets due to the fault effect. Hence, the existence of the fault is proved after $k=200$ since the intersection between the invariant sets that show the healthy operation of the system and the residual sets that are generated iteratively during the simulation can be found empty. Furthermore, the fault with this magnitude can be detected since $0 \notin\left\langle c_{r^{i o}}, b\left(R_{r^{i o}}\right)\right\rangle$. Thus, the fault alarm in Figure 8 gives the value 1 showing the existence of the fault. But, as it is explained previously, it can be observed that the FD decision is faster using interval observer-based approach in comparison with the set-invariance theory.
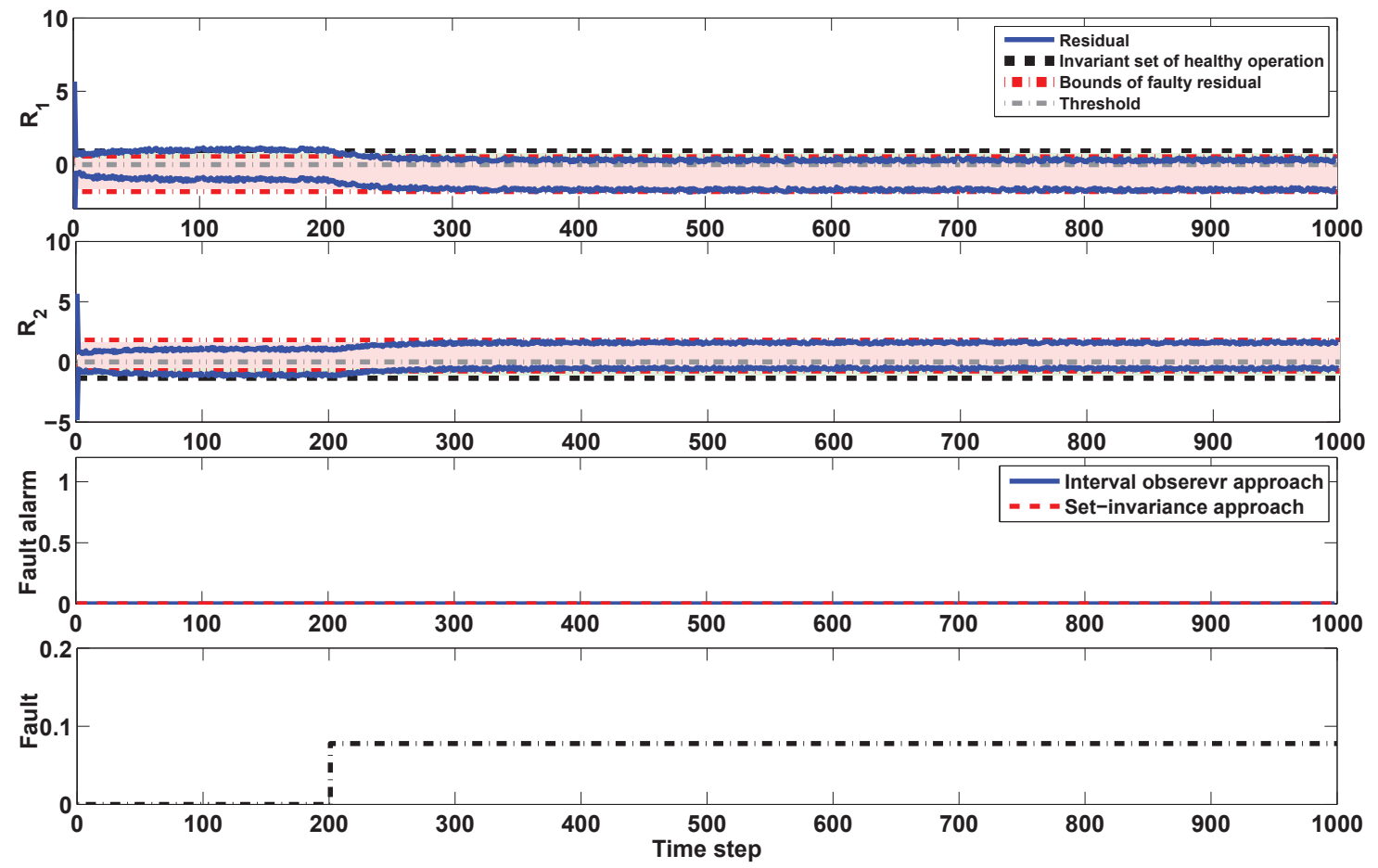

Figure 9.: FD test in the case of input sensor fault with the magnitude smaller than $0.0878 \mathrm{~V}$.

Additionally, the FD test is done when the fault occurs in the input of the observer with the magnitude slightly smaller than $0.0878 \mathrm{~V}$ and the result from the simulation is shown in Figure 9. This figure also shows that the residual sets are inside of the invariant sets at all time range. Therefore, this magnitude of the fault that is shown in bottom plot of Figure 9 is not detectable since neither Algorithm 1 nor (25) are satisfied. The overlap between the invariant sets and the residual sets emphasizes the issue that the fault can not be detected with the magnitude smaller than $0.0878 \mathrm{~V}$. The fault can never be detected if the overlap between the invariant set and the residual exists.

\subsection{Discussion}

This paper seeks to integrate the computation of the MDF using interval observer-based and setinvariance-based approaches undertaken by the classical sensitivity analysis. In order to detect the fault using both interval observer-based and set-invariance-based approaches, first the residual set in healthy operation of the system is obtained. Then, the residual (obtained in real-time) is compared with the healthy one. Furthermore, the FD test using both approaches is almost similar. In the interval observer-based approach, 0 is considered as the healthy threshold and the residual zonotope is calculated on-line. On the other hand, in the set-invariance approach, the healthy residual set (RPI set), which is computed off-line, is considered as healthy threshold. 
Since the FD principles can be integrated as it is introduced in Section 3.3, the obtained invariant set in steady state can be fixed as a set introducing the healthy operation of the system for both approaches. It means that the healthy zonotopic residual set using an interval observer in steady state, i.e., when $k \rightarrow \infty$, converges toward the healthy residual set computed using setinvariance approach. Then, assuming the initial state estimation error $\tilde{x}_{0}$ belongs to the zonotopic set $\tilde{\mathcal{X}}_{0}=\left\langle c_{\tilde{x}_{0}}, R_{\tilde{x}_{0}}\right\rangle$ that is defined as an RPI set, the corresponding residual set is computed as a zonotopic set using Proposition 3.4.

By understanding the possible way to ingrate the FD test using interval observer-based and set-invariance approaches as in Proposition 3.6, minimum detectable sensors and actuator faults are characterized in (45), (55) and (63) using the integrated FD principle, respectively. Using the classical sensitivity analysis, the MDF formulations in Section 4 is obtained considering the steady state operation of the system that lead to establish a bridge between set-invariance and interval observer-based approaches in FD framework. Further research is done in this section based on a case study to investigate the effectiveness of the obtained expressions for computing minimum magnitude of the fault that can be detected.

Three different mentioned fault scenarios in Section 4 are considered for the case study in order to test the obtained MDF formulations for the case of actuator and sensor faults. First, the magnitude of each type of fault that can be detected is computed theoretically based on the results presented Section 4. Then, the MDF magnitudes are compared with the one that are obtained form the simulation where the size of the fault is increased until the detectable magnitude for each case. Then, for the completion of the analysis, the FD test using the interval observer-based approach and setinvariance theory are simulated based on Algorithm 1 and (25) for both slightly bigger and slightly smaller magnitudes of the fault that are obtained theoretically in order to show the effectiveness and advantage of the interval observer in comparison with the set-invariance theory. As it can be observed from Figures 3, 6 and 8, both interval observer-based and set-invariance approaches are able to detect the fault whose the magnitudes are simulated bigger that the obtained MDF. But, the performance of the interval observer-based approach is faster in FD in comparison with setinvariance theory since its FD test is based on cheeking if $0 \notin\left\langle c_{r^{i o}}, b\left(R_{r^{i o}}\right)\right\rangle$. On the other hand, FD decision with set-invariance theory should be done by checking $r \notin\left\langle c_{r_{\infty}^{i s}}, R_{r_{\infty_{i}}^{i s}}\right\rangle$ that requires more time than interval observer-based approach. Faster decision about the existence of the fault can be understood as the advantage of interval observer-based approach in comparison with setinvariance theory. Furthermore, the FD test based on both approaches is also done considering the slightly smaller magnitudes that the obtained MDF. Figures 5, 7 and 9 show that the faults whose magnitudes are not detectable for the both interval observer-based and set-invariance approaches. Therefore, using sensitivity analysis with the integrated interval observer-based approach and setinvariance theory is well suited to also address the minimum magnitude of the fault that can be detectable for both interval observer-based and set-invariance approaches.

\section{Conclusion}

This paper has developed a study to characterize the Minimum Detectable Fault (MDF) for linear uncertain systems when the interval observer-based approach is used. Moreover, sensitivity analysis and set-invariance approach have been integrated for the Fault Detection (FD) purposes. Accordingly, the MDF has been characterized based on the sensitivity analysis integrated with setinvariance approach considering all the possible uncertainties in state and output measurement. Comparing both approaches yields to the same value of the MDF. Finally, a case study based on quadruple-tanks system is used to illustrate the obtained results. As a future research, the influence of the input over the magnitude of the MDF will be further analyzed in order to apply active diagnosis to design the system input such that both fault detection and isolation are able to be 
better performed. Furthermore, different faults will be considered based on their nature in order to characterize the minimum detectable incipient, multiplicative or intermittent faults.

\section{Appendix}

Definition 1: (Zonotope) A zonotope $\langle c, R\rangle \subset \mathbb{R}^{n}$ with the center $c \in \mathbb{R}^{n}$ and the generator matrix $R \in \mathbb{R}^{n \times p}$ is a polytopic set defined as a linear image of the unit hypercube $[-1,1]^{n}$, i.e.,

$$
\langle c, R\rangle=\left\{c+R s,\|s\|_{\infty} \leq 1\right\} .
$$

Moreover, a centered zonotope is denoted by $\langle R\rangle=\langle 0, R\rangle$. Any permutation of the columns of $R$ leaves it invariant.

Definition 2: (Minkowski sum) Considering two sets $\mathcal{A}$ and $\mathcal{B}$, their Minkowski sum is a set defined as $\mathcal{A} \oplus \mathcal{B}=\{a+b \mid a \in \mathcal{A}, b \in \mathcal{B}\}$. Furthermore, the Minkowski sum of the zonotopes $\mathcal{Z}_{1}=\left\langle c_{1}, R_{1}\right\rangle$ and $\mathcal{Z}_{2}=\left\langle c_{2}, R_{2}\right\rangle$ is $\mathcal{Z}_{1} \oplus \mathcal{Z}_{2}=\left\langle c_{1}+c_{2},\left[\begin{array}{ll}R_{1} & R_{2}\end{array}\right]\right\rangle$.

Definition 3: (Interval hull) The interval hull of a given zonotope $\mathcal{Z}=\langle c, R\rangle$, denoted by $\square \mathcal{Z}$, is the smallest interval box that contains $\mathcal{Z}$ and can be evaluated for all $i=1,2, \ldots, n$ as

$$
\square \mathcal{Z}=\left\{z:\left|z_{i}-c_{i}\right| \leq\left\|R_{i}\right\|_{1}\right\}
$$

where $R_{i}$ indicates the $i$-th line of matrix $R$, and $z_{i}$ and $c_{i}$ are the $i$-th components of $z$ and $c$, respectively.

Definition 4: (Invariant set) The invariant set $\Omega \subseteq \mathcal{Z}$ is the set which its existence allowed the evolution of a constrained system, where $z_{0} \in \Omega \subseteq \mathcal{Z}$ and then, $z_{K} \in \Omega \subseteq \mathcal{Z}$ for all time steps $k$.

Property 1: (Linear image) The linear image of a zonotope $\mathcal{Z}=\langle c, R\rangle$ by a compatible matrix $L$ is $L \odot\langle c, R\rangle=\langle L c, L R\rangle$.

Property 2: (Reduction operator) A reduction operator, denoted $\downarrow_{q}$, permits to reduce the number of generators of a zonotope $\langle c, R\rangle$ to a fixed number $q$ while preserving the inclusion property $\langle c, R\rangle \subset\left\langle c, \downarrow_{q}\{R\}\right\rangle$. A simple yet efficient solution to compute $\downarrow_{q}\{R\}$ is given in Combastel (2003). It consists in sorting the columns of $R$ on decreasing Euclidean norm and enclosing the influence of the smaller columns only into an easily computable interval hull, so that the resulting matrix $\downarrow_{q}\{R\}$ has no more than $q$ columns.

Property 3: (Zonotope inclusion) Given a zonotope $\mathcal{Z}=\langle c, R\rangle \subset \mathbb{R}^{n}$, a zonotope inclusion, indicated by $\diamond(\mathcal{Z})$, is defined as $\diamond(\mathcal{Z})=\langle c,[\operatorname{mid}(R) S]\rangle$, where $S$ is a diagonal matrix that satisfies $S_{i i}=\sum_{j=1}^{m} \frac{\operatorname{diam}\left(R_{i j}\right)}{2}, i=1,2, \cdots, n$, with $\operatorname{mid}($.$) and \operatorname{diam}($.$) being the center and$ diameter of the interval matrix, respectively.

Property 4: (State zonotope inclusion) Given $\mathcal{X}_{k+1}=A \mathcal{X}_{k} \oplus B u_{k}$, where $A$ and $B$ are interval matrices and $u_{k}$ is the input at time instant $k$, considering $\mathcal{X}_{k}$ as a zonotope with the center $c_{x, k}$ and the shape matrix $R_{x, k}$ such $\mathcal{X}_{k}=\left\langle c_{x, k}, R_{x, k}\right\rangle$, the zonotopic state at the next time instant $k+1$, 
defined as $\mathcal{X}_{k+1}$, is bounded by a zonotope $\mathcal{X}_{k+1}^{e}=\left\langle c_{x, k+1}, R_{x, k+1}\right\rangle$, with

$$
\begin{aligned}
c_{x, k+1} & =\operatorname{mid}(A) c_{x, k}+\operatorname{mid}(B) u_{k}, \\
R_{x, k+1} & =\left[\begin{array}{lll}
\diamond\left(A R_{x, k}\right) & \frac{\operatorname{diam}(A)}{2} c_{x, k} & \frac{\operatorname{diam}(B)}{2} u_{k}
\end{array}\right],
\end{aligned}
$$

where $\diamond\left(A R_{x, k}\right)$ shows the shape matrix of the state bounding zonotope.

\section{Acknowledgment}

This work has been partially funded by the Spanish Government (MINECO) through the project DEOCS (ref. DPI2016-76493-C3-3-R), by MINECO and FEDER through the project HARCRICS (ref. DPI2014-58104-R) and by Agència de Gestió d'Ajuts Universitaris i de Recerca.

\section{References}

Alamo, T., Bravo, J. M., \& Camacho, E. F. (2005). Guaranteed state estimation by zonotopes. Automatica, $41(6), 1035-1043$.

Artstein, Z., \& Raković, S. V. (2008). Feedback and invariance under uncertainty via set-iterates. Automatica, 44(2), 520-525.

Blanke, M., Kinnaert, M., Lunze, J., Staroswiecki, M., \& Schröder, J. (2006). Diagnosis and fault-tolerant control (Vol. 2). Springer, USA.

Cai, J., Ferdowsi, H., \& Sarangapani, J. (2016). Model-based fault detection, estimation, and prediction for a class of linear distributed parameter systems. Automatica, 66, 122-131.

Chai, W., \& Qiao, J. (2014). Passive robust fault detection using RBF neural modeling based on set membership identification. Engineering Applications of Artificial Intelligence, 28, 1-12.

Chen, J., \& Patton, R. J. (1999). Robust model-based fault diagnosis for dynamic systems. Kluwer Academic Press.

Chen, J., \& Patton, R. J. (2012). Robust model-based fault diagnosis for dynamic systems (Vol. 3). Springer Science \& Business Media.

Combastel, C. (2003). A state bounding observer based on zonotopes. In European Control Conference (ECC) (pp. 2589-2594).

Combastel, C. (2015). Zonotopes and Kalman observers: Gain optimality under distinct uncertainty paradigms and robust convergence. Automatica, 55, 265-273.

Ding, S. (2008). Model-based fault diagnosis techniques: design schemes, algorithms, and tools. Springer Science \& Business Media.

Fagarasan, I., Ploix, S., \& Gentil, S. (2004). Causal fault detection and isolation based on a set-membership approach. Automatica, 40(12), 2099-2110.

Gertler, J. (1998). Fault detection and diagnosis in engineering systems. USA: CRC Press.

Gertler, J. (2015). Fault detection and diagnosis. Springer.

Johansson, K. H. (2000). The quadruple-tank process: A multivariable laboratory process with an adjustable zero. IEEE Transactions on Control Systems Technology, 8(3), 456-465.

Kodakkadan, A. R., Pourasghar, M., Puig, V., Olaru, S., Ocampo-Martinez, C., \& Reppa, V. (2017). Observer-based sensor fault detectability: About robust positive invariance approach and residual sensitivity. In 20th world congress of the International Federation of Automatic Control (IFAC), France (pp. 5041-5046).

Kofman, E. (2005). Non-conservative ultimate bound estimation in LTI perturbed systems. Automatica, $41(10), 1835-1838$.

Kofman, E., Haimovich, H., \& Seron, M. M. (2007). A systematic method to obtain ultimate bounds for perturbed systems. International Journal of Control, 80(2), 167-178. 
Kolmanovsky, I., \& Gilbert, E. G. (1998). Theory and computation of disturbance invariant sets for discrete-time linear systems. Mathematical Problems in Engineering, 4(4), 317-367.

Lalami, A., \& Combastel, C. (2006). Generation of set membership tests for fault diagnosis and evaluation of their worst case sensitivity. In Fault Detection, Supervision and Safety of Technical Processes (SAFEPROCESS) (pp. 569-574). Elsevier.

Meseguer, J., Puig, V., Escobet, T., \& Saludes, J. (2010). Observer gain effect in linear interval observerbased fault detection. Journal of Process Control, 20(8), 944-956.

Ocampo-Martinez, C., De Doná, J. A., \& Seron, M. (2010). Actuator fault-tolerant control based on set separation. International Journal of Adaptive Control and Signal Processing, 24 (12), 1070-1090.

Pan, Y., \& Yang, G.-H. (2017). Event-triggered fault detection filter design for nonlinear networked systems. IEEE Transactions on Systems, Man, and Cybernetics: Systems.

Pourasghar, M., Puig, V., \& Ocampo-Martinez, C. (2016). Characterization of the minimum detectable fault of interval observers by using set-invariance theory. In 3rd conference on Control and Fault-Tolerant Systems (SysTol), Spain (pp. 79-86).

Puig, V. (2010). Fault diagnosis and fault tolerant control using set-membership approaches: Application to real case studies. International Journal of Applied Mathematics and Computer Science, 20(4), 619-635.

Puig, V., Montes de Oca, S., \& Blesa, J. (2013). Adaptive threshold generation in robust fault detection using interval models: time-domain and frequency-domain approaches. International Journal of Adaptive Control and Signal Processing, $27(10), 873-901$.

Puig, V., Quevedo, J., Escobet, T., \& de las Heras, S. (2002). Passive robust fault detection approaches using interval models. IFAC Proceedings Volumes, 35(1), 443-448.

Puig, V., Quevedo, J., Escobet, T., \& Stancu, A. (2003). Robust fault detection using linear interval observers. IFAC Proceedings Volumes, 36 (5), 579-584.

Raïssi, T., Efimov, D., \& Zolghadri, A. (2012). Interval state estimation for a class of nonlinear systems. IEEE Transactions on Automatic Control, 57(1), 260-265.

Rakovic, S. V., Kerrigan, E. C., Kouramas, K. I., \& Mayne, D. Q. (2005). Invariant approximations of the minimal robust positively invariant set. IEEE Transactions on Automatic Control, 50(3), 406-410.

Seron, M. M., \& De Doná, J. A. (2010). Actuator fault tolerant multi-controller scheme using set separation based diagnosis. International Journal of Control, 83(11), 2328-2339.

Seron, M. M., Zhuo, X. W., De Doná, J. A., \& Martínez, J. J. (2008). Multisensor switching control strategy with fault tolerance guarantees. Automatica, 44(1), 88-97.

Shen, B., Ding, S. X., \& Wang, Z. (2013). Finite-horizon $H_{\infty}$ fault estimation for linear discrete time-varying systems with delayed measurements. Automatica, 49(1), 293-296.

Silvestre, D., Rosa, P., Hespanha, J. P., \& Silvestre, C. (2017). Fault detection for LPV systems using set-valued observers: A coprime factorization approach. Systems $\&$ Control Letters, 106, 32-39.

Stoican, F., Hovd, M., \& Olaru, S. (2013). Explicit invariant approximation of the mRPI set for LTI dynamics with zonotopic disturbances. In 52nd Annual Conference on Decision and Control (CDC) (pp. $3237-3242)$.

Tabatabaeipour, S. M. (2015). Active fault detection and isolation of discrete-time linear time-varying systems: a set-membership approach. International Journal of Systems Science, 46(11), 1917-1933.

Tabatabaeipour, S. M., \& Bak, T. (2014). Robust observer-based fault estimation and accommodation of discrete-time piecewise linear systems. Journal of the Franklin Institute, 351 (1), 277-295.

Tan, C., Tao, G., \& Qi, R. (2013). A discrete-time parameter estimation based adaptive actuator failure compensation control scheme. International Journal of Control, 86(2), 276-289.

Thabet, R. E. H., Combastel, C., Raïssi, T., \& Zolghadri, A. (2015). Set-membership fault detection under noisy environment with application to the detection of abnormal aircraft control surface positions. International Journal of Control, 88(9), 1878-1894.

Uusitalo, L., Lehikoinen, A., Helle, I., \& Myrberg, K. (2015). An overview of methods to evaluate uncertainty of deterministic models in decision support. Environmental Modelling \& Software, 63, 24-31.

Xu, F., Puig, V., Ocampo-Martinez, C., Olaru, S., \& Stoican, F. (2015). Set-theoretic methods in robust detection and isolation of sensor faults. International Journal of Systems Science, 46(13), 2317-2334.

Xu, F., Stoican, F., Puig, V., Ocampo-Martinez, C., \& Olaru, S. (2013). On the relationship between interval observers and invariant sets in fault detection. In Control and Fault-Tolerant Systems (SysTol) (pp. 49-54).

Xu, F., Tan, J., Wang, X., Puig, V., Liang, B., Yuan, B., \& Liu, H. (2017). Generalized set-theoretic 
unknown input observer for LPV systems with application to state estimation and robust fault detection. International Journal of Robust and Nonlinear Control, 27(17), 3812-3832.

Zhong, M., Ding, S. X., Lam, J., \& Wang, H. (2003). An LMI approach to design robust fault detection filter for uncertain LTI systems. Automatica, 39(3), 543-550. 